\title{
Photochemically driven tandem process in the construction of a biscyclopropylcage from 2,5-dimethoxy-p-benzoquinone and terminal acetylenes
}

Jonathan Álvarez-García, ${ }^{a}$ Víctor Rubio-Pisabarro, ${ }^{a}$ Carlos Silva-López ${ }^{\text {a }}$ and María Magdalena Cid*a

\section{Index}

1. General Methods.............................................................................................................

2. General procedure for the [2+2] photoaddition of 2,5-dimethoxy- $p$ benzoquinone and alkyne ...................................................................................................

3. Substrates characterization.......................................................................................S3

4. Detailed Computational Methods.......................................................................................S19

5. Reaction profile with exo coordinates. $\quad$ S21

6. Cartesian coordinates S22

7. References ................................................................................................................ S43 


\section{General Methods}

All reactions were carried out under nitrogen atmosphere. 2,5-Dimethoxy-p-benzoquinone was synthesized from commercial 2,5-dihydroxy-p-benzoquinone according to the method reported in the literature. ${ }^{1}$ All other reagents were commercial compounds of the highest purity available. Thin-layer chromatography (TLC) was conducted on chromatoplates of $\mathrm{SiO}_{2} 60 \mathrm{~F}-254$ obtained from Merck and visualised by UV irradiation ( $254 \mathrm{~nm}$ ) or by staining with a solution of phosphomolibdic acid or vanillin. Flash chromatography was carried out with $\mathrm{SiO}_{2} 60$ (230-400 mesh; Merck) under pressure. ${ }^{1} \mathrm{H}-\mathrm{NMR}$ spectra were recorded at $25{ }^{\circ} \mathrm{C}$ on Bruker $\mathrm{AMX}-400$ at $400 \mathrm{MHz}$ with residual protic solvent as internal reference $\left[\mathrm{CDCl}_{3}, \delta_{\mathrm{H}}=7.26 \mathrm{ppm}\right]$, chemical shifts $(\delta)$ are given in parts per million (ppm) and coupling constants $(J)$ are given in Hertz $(\mathrm{Hz})$. The proton spectra are reported as follows: chemical shift $\delta$ (multiplicity, coupling constant $J$, number of protons). The following symbols were used for the description of coupling patterns: multiplet $(m)$, singlet $(s)$, doublet $(d)$, triplet $(t) .{ }^{13} \mathrm{C}$-NMR spectra were recorded on the same spectrometer at $100 \mathrm{MHz}$, with the central peak of $\mathrm{CDCl}_{3}, \delta_{\mathrm{C}}=77.16 \mathrm{ppm}$ as reference. UV-Vis spectra were recorded on a Jasco J-815 spectropolarimeter using a one-centimetre thick quartz cuvette at $25^{\circ} \mathrm{C}$. All compound solutions were prepared in acetonitrile by dissolving a known mass in a known volume of solvent. The background was always obtained against the solvent. IR spectra were recorded on a Nicolet 6700 IR-Turbo infrared spectrometer. Peaks are quoted in wavenumber $\left(\mathrm{cm}^{-1}\right)$. ESI mass spectra were recorded with an APEX3 instrument. Ions were generated using a Combi MALDI-electrospray ionization (ESI) source. Samples were prepared by adding a spray solution of 70:29:9:0.1 $(\mathrm{v} / \mathrm{v} / \mathrm{v}) \mathrm{CH}_{3} \mathrm{OH} /$ water/formic acid to a solution of the sample at a $\mathrm{v} / \mathrm{v}$ ratio of 1 to $5 \%$ to give the best signal-to-noise ratio. High-resolution mass spectra were taken on a VG Autospec instrument. Crystallographic data were collected at $100 \mathrm{~K}$ using a Bruker D8 Venture diffractometer with a Photon 100 CMOS detector and Mo-K $\alpha$ radiation $(\lambda=0.71073 \AA$ ) generated by an Incoatec high brillance microfocus source equipped with Incoatec Helios multilayer optics. The software APEX32 was used for collecting frames of data, indexing reflections, and the determination of lattice parameters, SAINT ${ }^{3}$ for integration of intensity of reflections, and $\mathrm{SADABS}^{4}$ for scaling and empirical absorption correction. Using OLEX $2^{5}$, the structure was solved by dual-space algorithm using the program SHELXT ${ }^{6}$ and all non-hydrogen atoms was refined with anisotropic thermal parameters by full-matrix least-squares calculations on $F^{2}$ with the SHELXL ${ }^{6}$ refinement package. Hydrogen atoms were inserted at calculated positions and constrained with isotropic thermal parameters. Drawings were produced with PLATON ${ }^{7}$. 


\section{General procedure for the $[2+2]$ photoaddition of 2,5-dimethoxy-p- benzoquinone and alkyne}

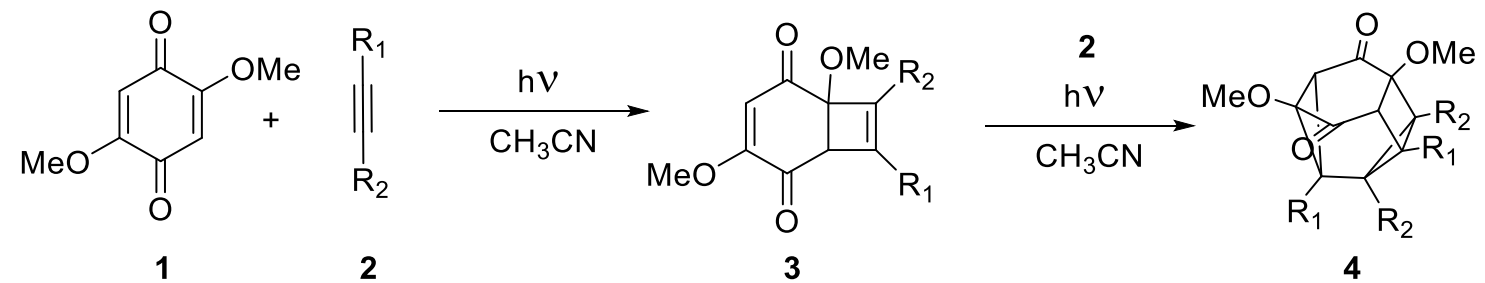

A suspension of 2,5-dimethoxy-p-benzoquinone 1 (1 equiv.) in acetonitrile $(0.03 \mathrm{M})$ in a Pyrex Schlenk flask provided with magnetic stirring was bubbled with $\mathrm{N}_{2}$ for 20 minutes. Then, the corresponding alkyne $\mathbf{2}$ ( 9 equiv.) was added and the reaction mixture was irradiated for $11 \mathrm{~h}$ * at room temperature, placing the reaction vessel about $10 \mathrm{~cm}$ from the irradiation source [Hanovia UV lamp $(450 \mathrm{~W})$ ]. The solvent was removed under reduced pressure and the remaining solid was purified by flash chromatography $\left(\mathrm{SiO}_{2}\right)$ using different hexane-AcOEt mixtures as solvent. * Unless otherwise stated.

\section{Substrates characterization}

1,4-dimethoxy-8-phenylbicyclo[4.2.0]octa-3,7-diene-2,5-dione (3a) and 3,7-dimethoxy-1,2diphenyl pentacyclo[4.4.0.0 $\left.0^{3,9} \cdot 0^{5,7} \cdot 0^{2,10}\right]$ decan-4,8-dione (4a)<smiles>COC1C(=O)CC2(OC)C=CC(=O)C12c1ccccc1</smiles>

3a

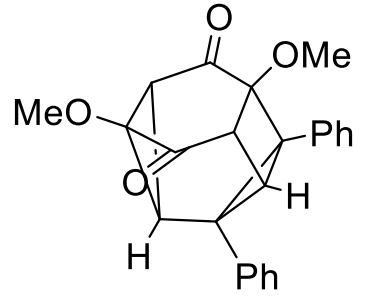

4a

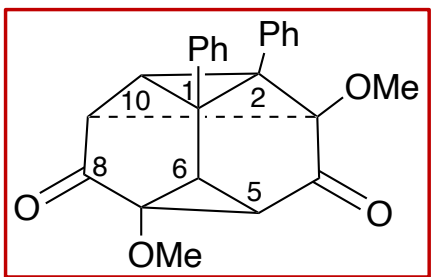

Following the general procedure, the reaction of $1(100 \mathrm{mg}, 0.595 \mathrm{mmol})$ and alkyne $2 \mathrm{a}(0.6 \mathrm{~mL}$, $5.35 \mathrm{mmol}$ ) rendered $3 \mathrm{a}$ as a yellowish solid ( $8 \mathrm{mg}, 5 \%$ ) and $4 \mathrm{a}$ as a white solid (89 $\mathrm{mg}, 40 \%$ ) after purification by flash chromatography $\left(\mathrm{SiO}_{2} ;\right.$ Hexane:AcOEt (8:2)).

When 1 (300 mg, $1.78 \mathrm{mmol})$ in acetonitrile $(60 \mathrm{~mL}$ ) was reacted with phenylacetylene 2a (1.8 $\mathrm{mL}, 16.4 \mathrm{mmol}$ ) for $12 \mathrm{~h}, \mathbf{4 a}$ was obtained as the only product ( $327 \mathrm{mg}, 49 \%$ ). $4 \mathrm{a}$ is thermally stable as shown when a toluene solution was refluxed for several hours and starts to degrade after prolonged irradiations times.

3a: ${ }^{1} \mathrm{H}-\mathrm{NMR}\left(400 \mathrm{MHz}, \mathrm{CDCl}_{3}\right): \delta=7.61(\mathrm{~m}, 2 \mathrm{H}), 7.35(\mathrm{~m}, 3 \mathrm{H}), 6.71(\mathrm{~d}, J=1.8 \mathrm{~Hz}, 1 \mathrm{H}), 5.98(\mathrm{~s}, 1 \mathrm{H})$, $3.97(\mathrm{~d}, J=1.8 \mathrm{~Hz}, 1 \mathrm{H}), 3.79(\mathrm{~s}, 3 \mathrm{H}), 3.41(\mathrm{~s}, 3 \mathrm{H}) .{ }^{13} \mathrm{C}-\mathrm{NMR}\left(100 \mathrm{MHz}, \mathrm{CDCl}_{3}\right): \delta=194.9,191.6$, 159.9, 151.7, 130.5, 130.3, 129.8, 128.8, 126.5, 111.1, 85.0, 56.7, 53.8, 53.4. HRMS (ESI-TOF) $\mathrm{m} / \mathrm{z}$ : $[\mathrm{M}+\mathrm{H}]^{+}$Calcd. for $\mathrm{C}_{16} \mathrm{H}_{15} \mathrm{O}_{4}$ 271.0965; Found 271.0960. UV-vis $\left(\mathrm{CH}_{3} \mathrm{CN}\right): \lambda(\varepsilon): 254$ (33044 $\left.\mathrm{Lmol}^{-1} \mathrm{~cm}^{-1}\right), 346\left(2566 \mathrm{Lmol}^{-1} \mathrm{~cm}^{-1}\right) \mathrm{nm}$. FT-IR (ATR) v 3442, 3429, 3409, 3396, 3382, 3303, 3062 , 3022, 2954, 2924, 2853, 1997,1702, 1663, 1602, 1554, 1492, 1449, 1362, 1305, 1254, 1239, 
1186, 1166, 1099, 1081, 1012, 987, 961, 928, 854, 806, 767, 744, 694, 643, 590, 537, 491, 449 $\mathrm{cm}^{-1}$.

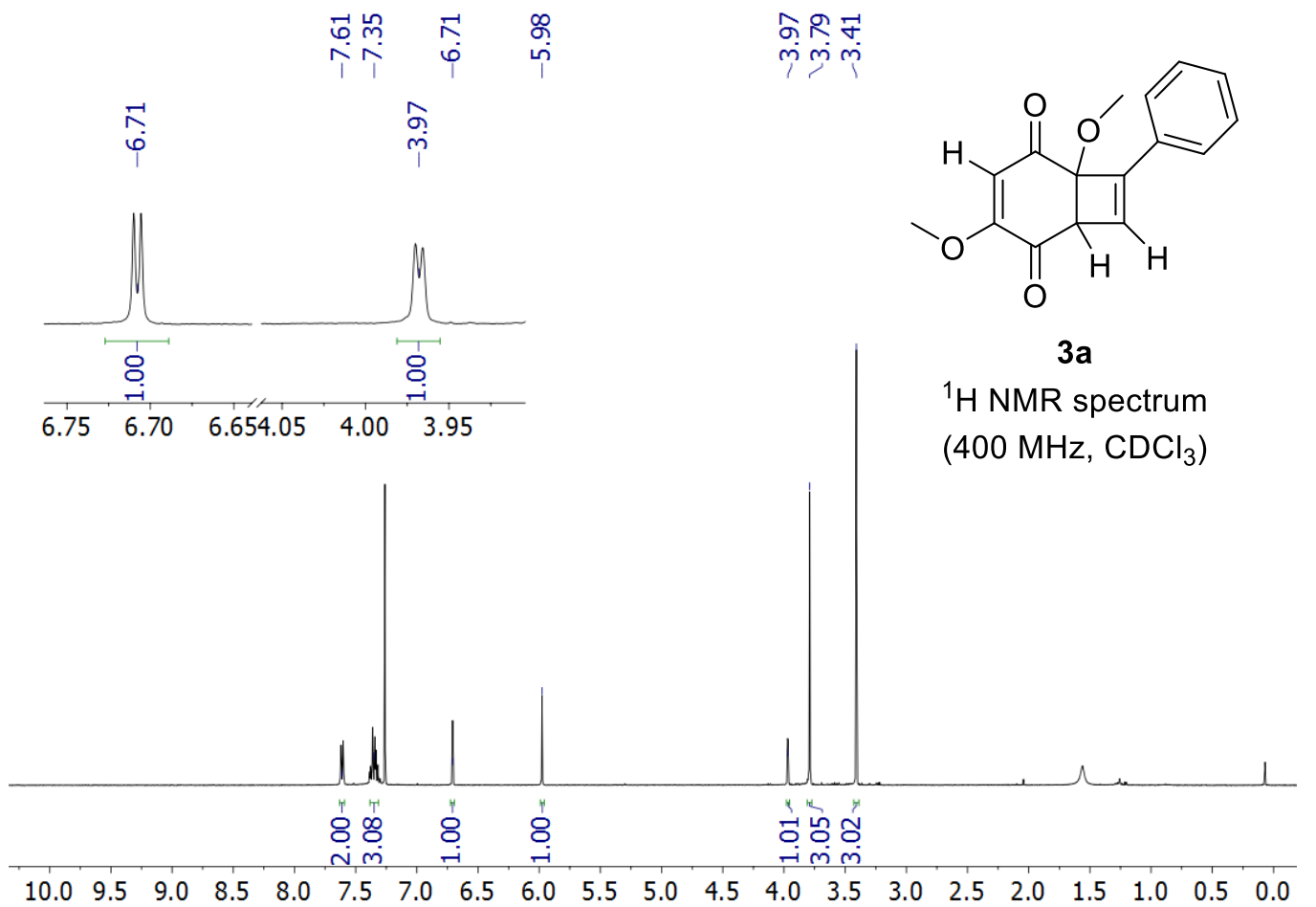

Figure S1. $3 a^{1} \mathrm{H}-\mathrm{NMR}\left(\mathrm{CDCl}_{3}\right)$ spectra.
$œ$ in
ஓั
नำ 유요 \&

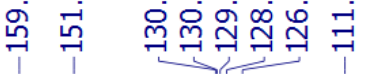<smiles>COC1C(=O)C=CC2(OC)C=CC(=O)C12c1ccccc1</smiles>

3a

${ }^{13} \mathrm{C}$ NMR spectrum $\left(100 \mathrm{MHz}, \mathrm{CDCl}_{3}\right)$

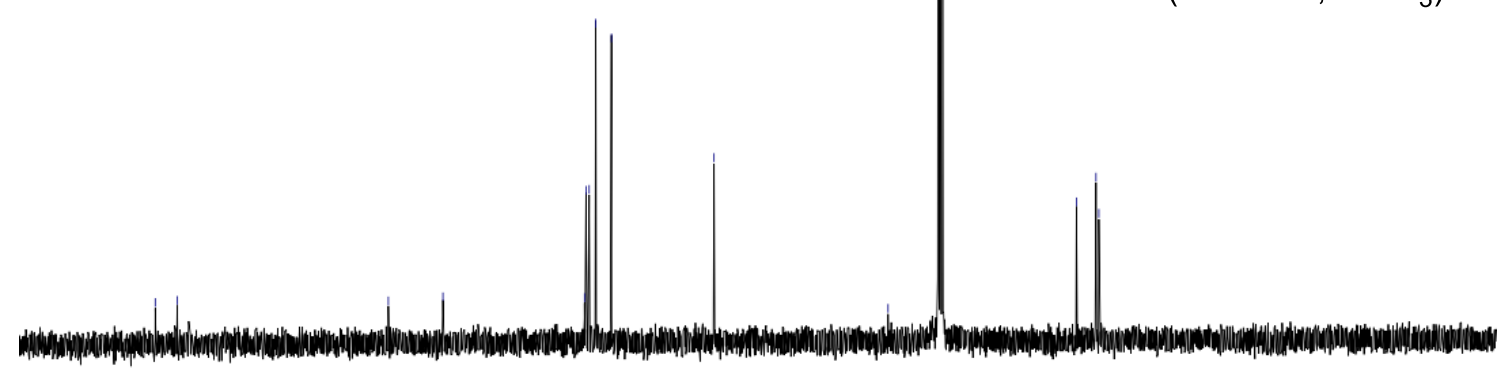

$\begin{array}{llllllllllllllllllllll}210 & 200 & 190 & 180 & 170 & 160 & 150 & 140 & 130 & 120 & 110 & 100 & 90 & 80 & 70 & 60 & 50 & 40 & 30 & 20 & 10 & 0\end{array}$

Figure S2. $3 a{ }^{13} \mathrm{C}-\mathrm{NMR}\left(\mathrm{CDCl}_{3}\right)$ spectra. 


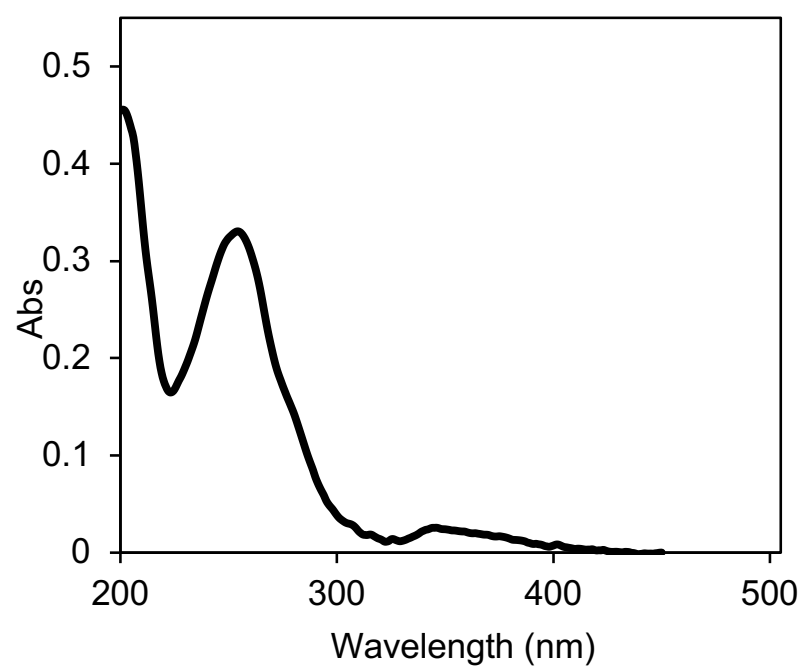

Figure S3. UV/Vis spectra of $3 a\left[1.0 \cdot 10^{-5} \mathrm{M}\right]$ in acetonitrile.

4a: ${ }^{1} \mathrm{H}-\mathrm{NMR}\left(400 \mathrm{MHz}, \mathrm{CDCl}_{3}\right): \delta=7.06(\mathrm{~m}, 8 \mathrm{H}), 6.65(\mathrm{~m}, 2 \mathrm{H}), 3.74(\mathrm{~d}, J=5.0 \mathrm{~Hz}, 1 \mathrm{H}), 3.54(\mathrm{~s}, 3 \mathrm{H})$, 3.31 (s, 3H), 3.27 (d, J=5.0 Hz, 1H), 3.25 (d, J=10 Hz, 1H), 2.85 (d, J=10 Hz, 1H). ${ }^{13} \mathrm{C}-\mathrm{NMR}(100$ $\left.\mathrm{MHz}_{\mathrm{CDCl}}\right): \delta=202.1,200.4,136.9,131.7,129.7,128.8,127.9,127.8,127.7,127.1,82.9,74.1$, 58.0, 53.0, 52.9, 50.4, 45.0, 44.6, 37.6, 36.7. HRMS (ESI-TOF) $\mathrm{m} / \mathrm{z}$ : $[\mathrm{M}+\mathrm{H}]^{+}$Calcd. for $\mathrm{C}_{24} \mathrm{H}_{21} \mathrm{O}_{4}$ 373.1434; Found 373.1430. UV-vis $\left(\mathrm{CH}_{3} \mathrm{CN}\right): \lambda(\varepsilon): 208\left(31889 \mathrm{Lmol}^{-1} \mathrm{~cm}^{-1}\right), 259\left(1358 \mathrm{Lmol}^{-1} \mathrm{~cm}^{-}\right.$ $\left.{ }^{1}\right), 315\left(571 \mathrm{Lmol}^{-1} \mathrm{~cm}^{-1}\right) \mathrm{nm}$. FT-IR (ATR) $\vee$ 3028, 2916, 2848, 2726, 2641, 1739, 1714, 1682, $1506,1468,1371,1295,1238,1177,1131,1070,1044,1020,984,932,890,757,719,631,608$ $\mathrm{cm}^{-1}$.

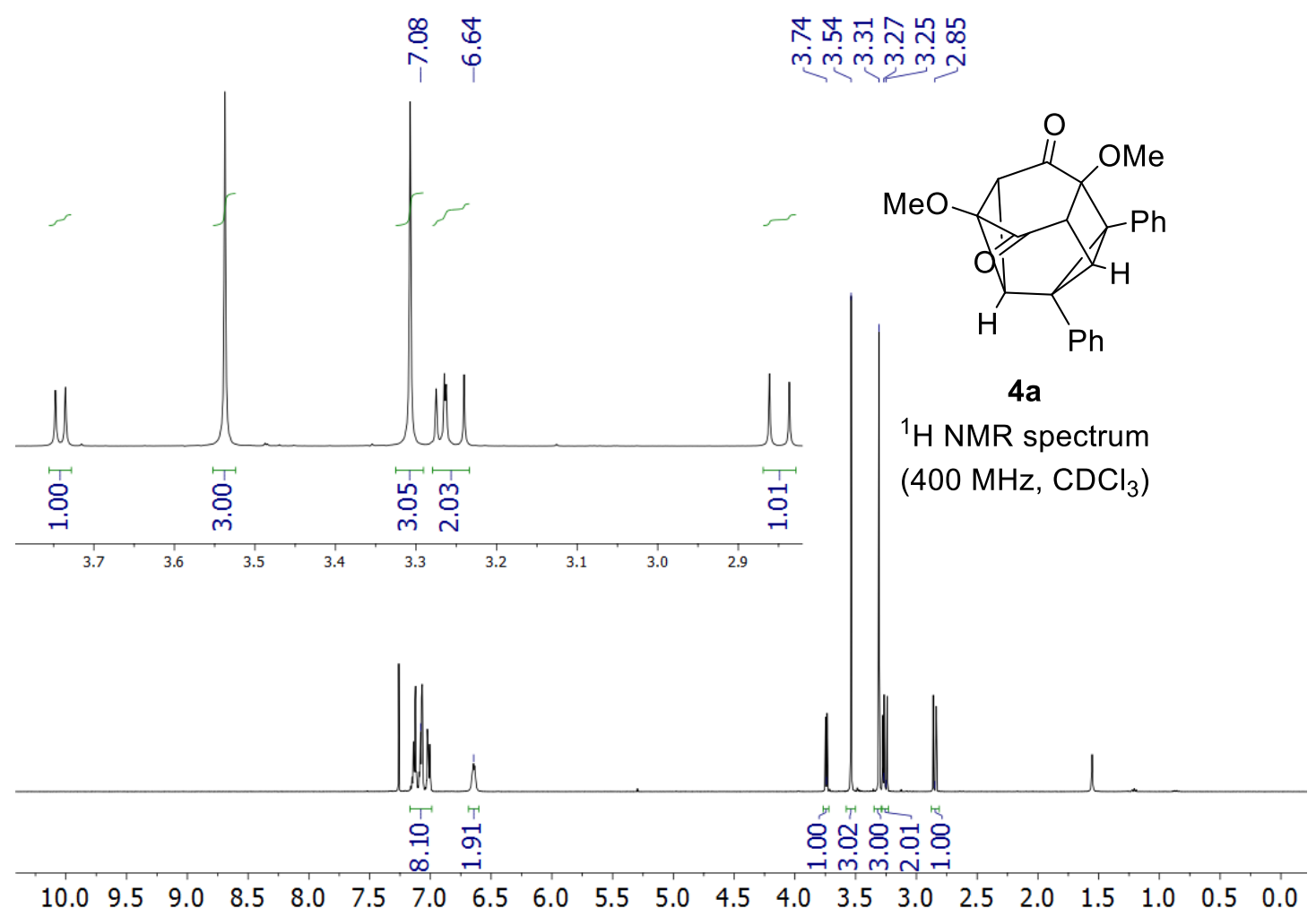


Figure S4. 4a ${ }^{1} \mathrm{H}-\mathrm{NMR}\left(\mathrm{CDCl}_{3}\right)$ spectra.
必 는
๓
్ㅠㅇㅠ

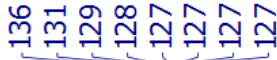

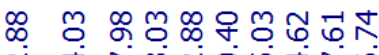

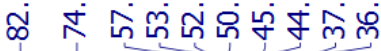

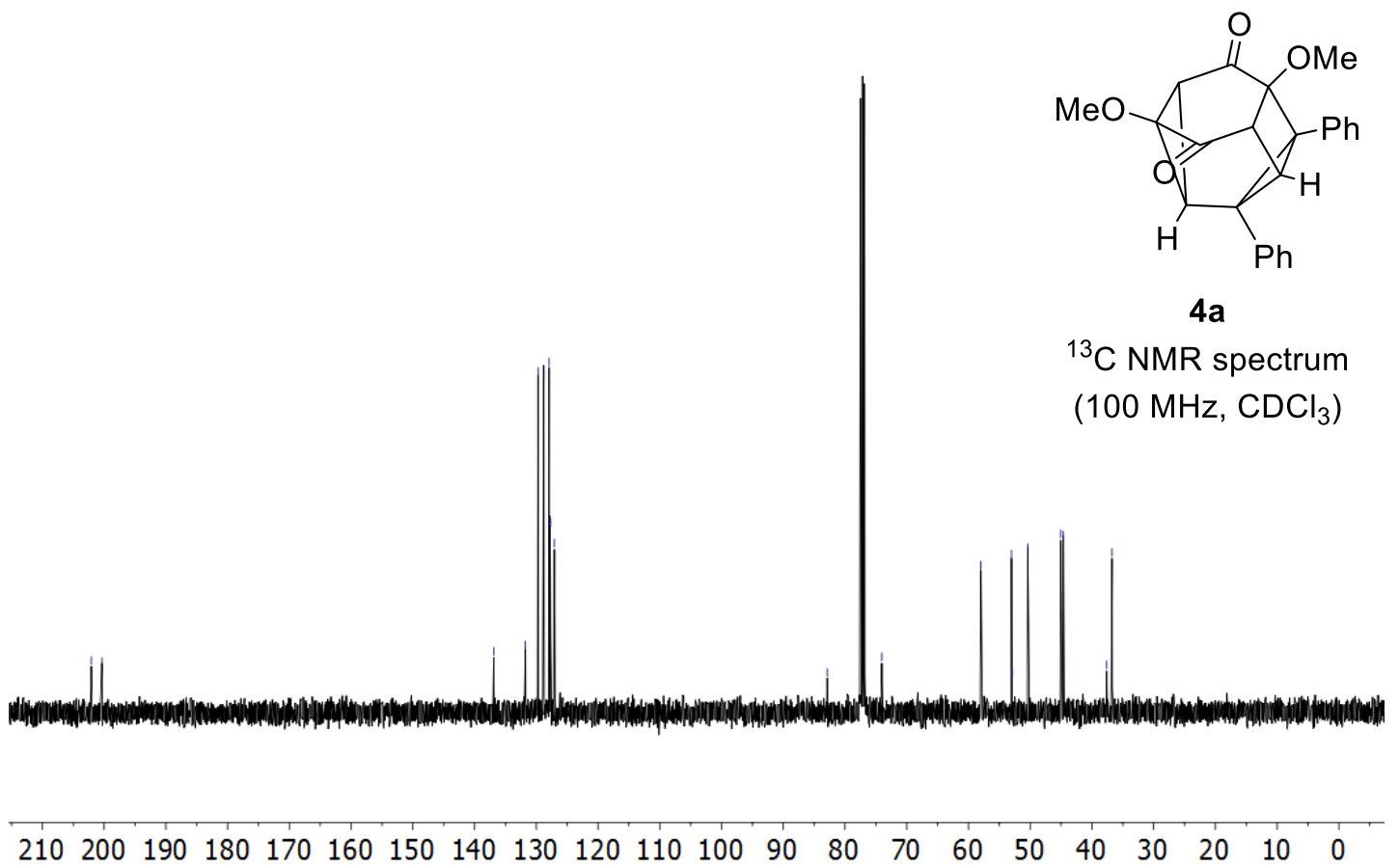

Figure S5. $4 a{ }^{13} \mathrm{C}-\mathrm{NMR}\left(\mathrm{CDCl}_{3}\right)$ spectra.

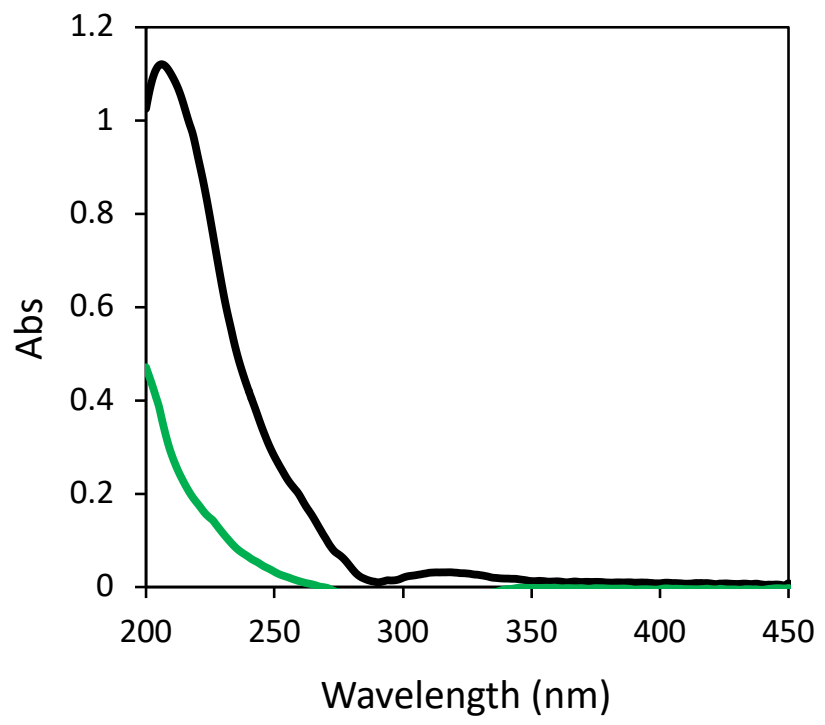

Figure S6. UV/Vis spectra of $4 \mathrm{a}$ in acetonitrile: black line $\left[5.5 \cdot 10^{-5} \mathrm{M}\right]$ and green line $\left[1.0 \cdot 10^{-5} \mathrm{M}\right]$. 


\section{Crystallographic data of $4 a$}

Crystals suitable for $x$-ray diffraction of compound $4 a$ can be grown from the slow evaporation of a solution of $4 a$ in a hexane:ethyl ether(1:1) mixture.
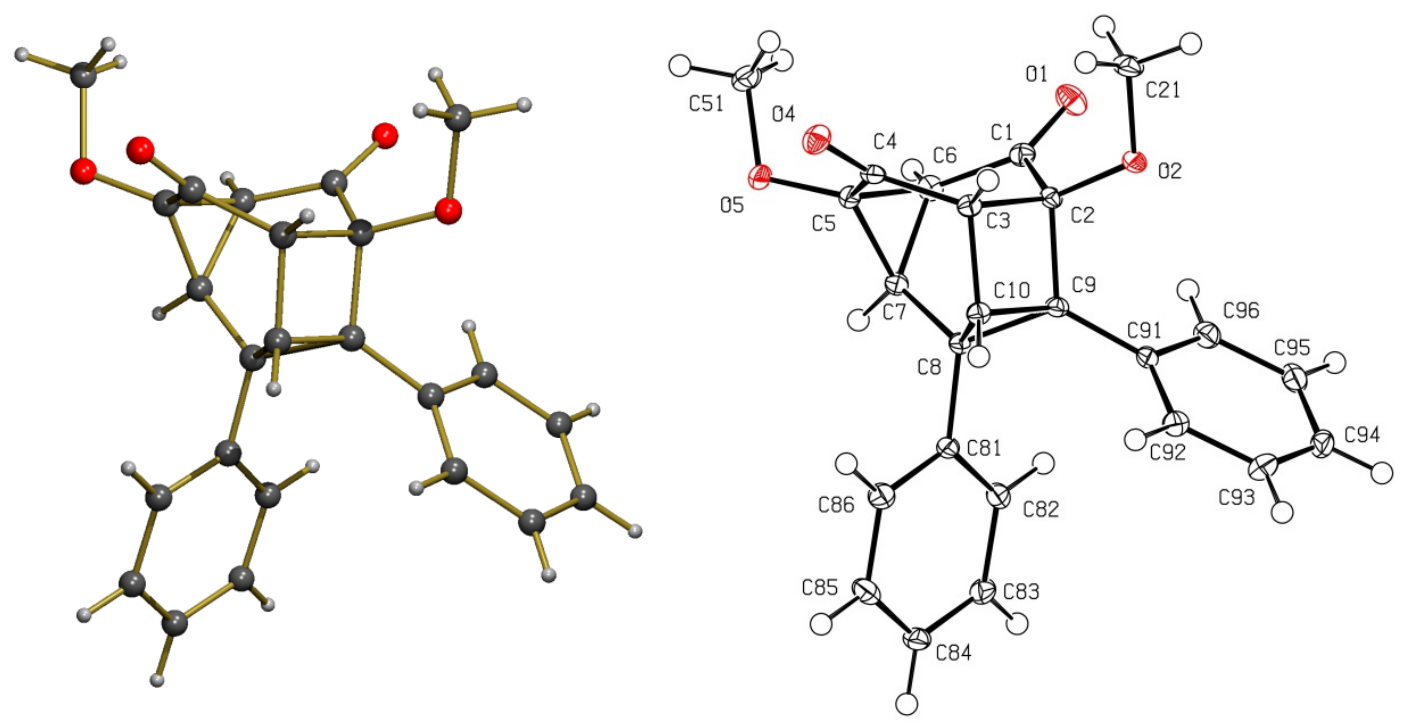

Figure S7. 4a ORTEP type diagram (ellipsoid at 50\% probability) .

Table S1. Crystal data and structure refinement for 4a. (CCDC 2000238)

Identification code

Empirical formula

Formula weight

Temperature

Wavelength

Crystal system

Space group

Unit cell dimensions

Volume

Z

Density (calculated)

Absorption coefficient

$\mathrm{F}(000)$

Crystal size

Theta range for data collection

Index ranges 32041901_0m

C24 H20 O4

372.40

$100.01 \mathrm{~K}$

$0.71073 \AA$

Monoclinic

P 1 21/c 1

$\begin{array}{ll}\mathrm{a}=11.7790(5) \AA & \alpha=90^{\circ} . \\ \mathrm{b}=6.2989(3) \AA & \beta=95.942(2)^{\circ} . \\ \mathrm{c}=24.9390(11) \AA & \gamma=90^{\circ} .\end{array}$

1840.40(14) $\AA^{3}$

4

$1.344 \mathrm{Mg} / \mathrm{m}^{3}$

$0.091 \mathrm{~mm}^{-1}$

784

$0.112 \times 0.091 \times 0.036 \mathrm{~mm}^{3}$

2.264 to $28.341^{\circ}$.

$-15<=\mathrm{h}<=12,-8<=\mathrm{k}<=8,-33<=\mathrm{l}<=33$ 
Reflections collected

Independent reflections

Completeness to theta $=25.242^{\circ}$

Absorption correction

Max. and min. transmission

Refinement method

Data / restraints / parameters

Goodness-of-fit on $\mathrm{F}^{2}$

Final $\mathrm{R}$ indices $[\mathrm{I}>2 \operatorname{sigma}(\mathrm{I})]$

$\mathrm{R}$ indices (all data)

Extinction coefficient

Largest diff. peak and hole
34487

$4587[\mathrm{R}(\mathrm{int})=0.0412]$

$99.9 \%$

Semi-empirical from equivalents

0.7457 and 0.6888

Full-matrix least-squares on $\mathrm{F}^{2}$

4587 / 0 / 255

1.015

$\mathrm{R} 1=0.0427, \mathrm{wR} 2=0.1020$

$\mathrm{R} 1=0.0552, \mathrm{wR} 2=0.1089$

$\mathrm{n} / \mathrm{a}$

0.432 and -0.273 e. $\AA^{-3}$

1,4-Dimethoxy-8-phenylbicyclo[4.2.0]octa-3,7-diene-2,5-dione-7-d biscyclopropylcage (4aD)<smiles>[2H]C1=C(c2ccccc2)C2(OC)C(=O)C=C(OC)C(=O)C12</smiles>

3aD

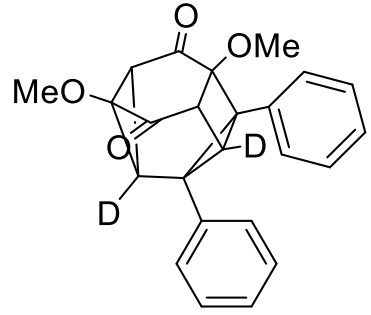

4aD

2aD was synthesized according to methods reported in the literature. ${ }^{8}$ Following the general procedure, the reaction of $1(100 \mathrm{mg}, 0.595 \mathrm{mmol})$ and alkyne 2aD (0.6 mL, $5.35 \mathrm{mmol})$ rendered $3 \mathrm{aD}$ as a yellowish solid ( $7 \mathrm{mg}, 4 \%$ ) and $4 \mathrm{aD}$ as a white solid (82 $\mathrm{mg}, 37 \%$ ) after purification by flash chromatography $\left(\mathrm{SiO}_{2}\right.$; Hexane:AcOEt (8:2)). 
3aD : ${ }^{1} \mathrm{H}-\mathrm{NMR}\left(400 \mathrm{MHz}, \mathrm{CDCl}_{3}\right): \delta=7.61(\mathrm{~m}, 2 \mathrm{H}), 7.34(\mathrm{~m}, 3 \mathrm{H}), 5.98(\mathrm{~s}, 1 \mathrm{H}), 3.97(\mathrm{~s}, 1 \mathrm{H}), 3.79(\mathrm{~s}$, $3 \mathrm{H}), 3.39(\mathrm{~s}, 3 \mathrm{H})$.

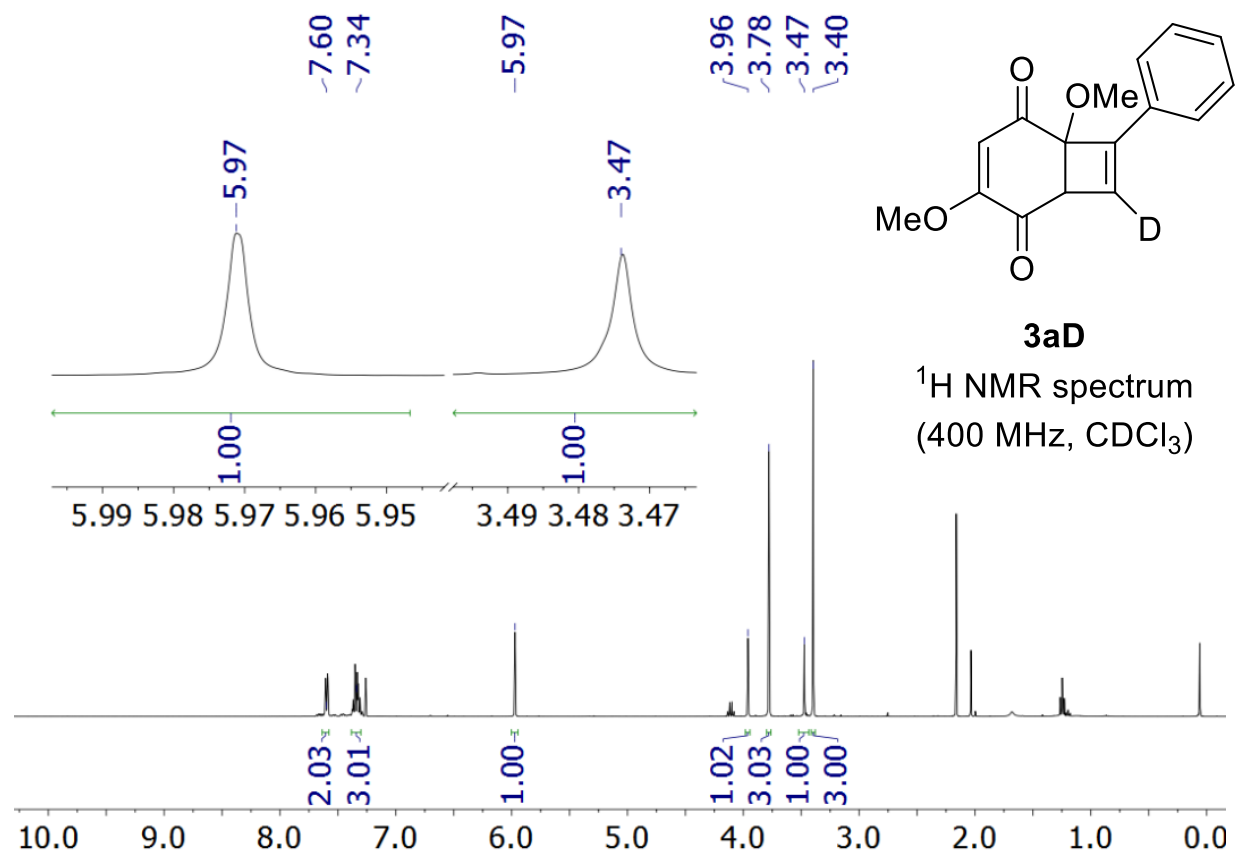

Figure S8. $3 a(D){ }^{1} \mathrm{H}-\mathrm{NMR}\left(\mathrm{CDCl}_{3}\right)$ spectra.

4aD : ${ }^{1} \mathrm{H}-\mathrm{NMR}\left(400 \mathrm{MHz}, \mathrm{CDCl}_{3}\right): \delta=7.06(\mathrm{~m}, 8 \mathrm{H}), 6.65(\mathrm{~m}, 2 \mathrm{H}), 3.53(\mathrm{~s}, 3 \mathrm{H}), 3.29(\mathrm{~s}, 3 \mathrm{H}), 3.26(\mathrm{~s}$, $1 \mathrm{H}), 2.84(\mathrm{~s}, 1 \mathrm{H})$.

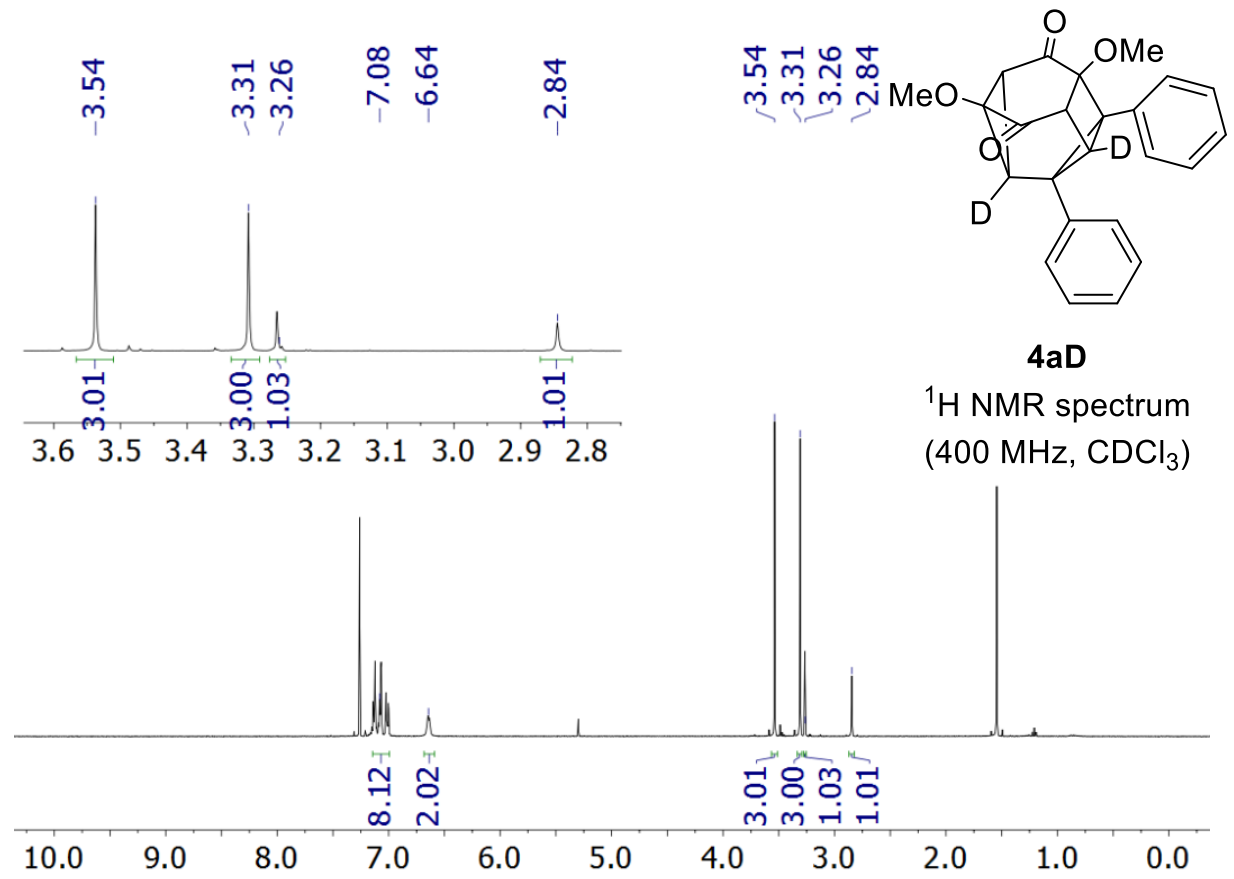

Figure S9. $4 \mathrm{aD}{ }^{1} \mathrm{H}-\mathrm{NMR}\left(\mathrm{CDCl}_{3}\right)$ spectra. 
<smiles>COC1=CC(=O)C2(OC)C(=O)C(c3ccccc3)=C(c3ccccc3)C2C1=O</smiles>

3b

Following the general procedure, the reaction of $1(100 \mathrm{mg}, 0.595 \mathrm{mmol})$ and alkyne $\mathbf{2 b}(0.95 \mathrm{~g}$, $5.35 \mathrm{mmol}$ ) rendered $\mathbf{3 b}$ as a yellowish solid $(16 \mathrm{mg}, 8 \%)$ after purification by flash chromatography $\left(\mathrm{SiO}_{2} ;\right.$ Hexane:AcOEt (7:3)).

3b: ${ }^{1}$ H-NMR (400 MHz, $\left.\mathrm{CDCl}_{3}\right): \delta=7.72(\mathrm{~m}, 2 \mathrm{H}), 7.58(\mathrm{~m}, 2 \mathrm{H}), 7.33(\mathrm{~m}, 6 \mathrm{H}), 6.02(\mathrm{~s}, 1 \mathrm{H}), 4.32(\mathrm{~s}$, 1H), $3.74(\mathrm{~s}, 3 \mathrm{H}), 3.40$ (s, 3H). ${ }^{13} \mathrm{C}-\mathrm{NMR}\left(100 \mathrm{MHz}, \mathrm{CDCl}_{3}\right): \delta=195.1,191.6,160.2,142.8,142.2$, $132.2,131.5,129.9,129.4,128.9,128.8,127.6,127.5,111.1,83.0,56.7,55.4,53.4$. HRMS (ESITOF) $\mathrm{m} / \mathrm{z}:[\mathrm{M}+\mathrm{H}]^{+}$Calcd. for $\mathrm{C}_{22} \mathrm{H}_{19} \mathrm{O}_{4}$ 347.1278; Found 347.1279. UV-vis $\left(\mathrm{CH}_{3} \mathrm{CN}\right): \lambda(\varepsilon): 198$ (55961 $\left.\mathrm{Lmol}^{-1} \mathrm{~cm}^{-1}\right), 256\left(7410 \mathrm{Lmol}^{-1} \mathrm{~cm}^{-1}\right) \mathrm{nm}$. FT-IR (ATR) $v 3497,3445,3406,3380,3056$, 3027, 2983, 2936, 2851, 1829, 1702,1663, 1575, 1541, 1488, 1458, 1445, 1361, 1329, 1298, $1239,1188,1165,1114,1095,1080,1029,1010,980,935,868,844,802,771,759,720,691 \mathrm{~cm}^{-}$ 1.

\begin{tabular}{|c|c|c|}
\hline 卡品品 & $\underset{0}{\stackrel{D}{0}}$ & $\underset{\sim}{\tilde{\sim}}$ \\
\hline
\end{tabular}

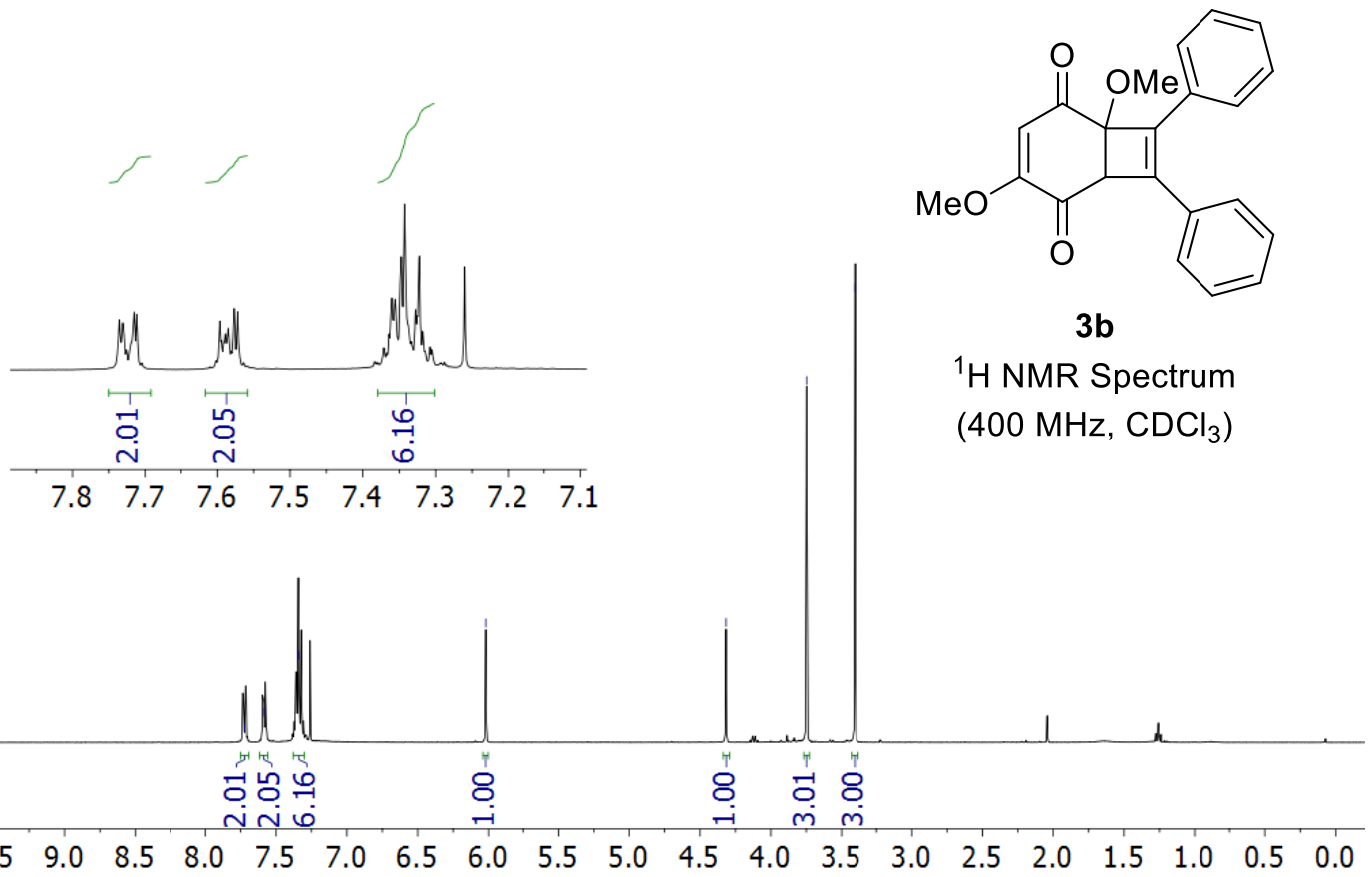

Figure S10. $3 \mathbf{b}^{1} \mathrm{H}-\mathrm{NMR}\left(\mathrm{CDCl}_{3}\right)$ spectra. 


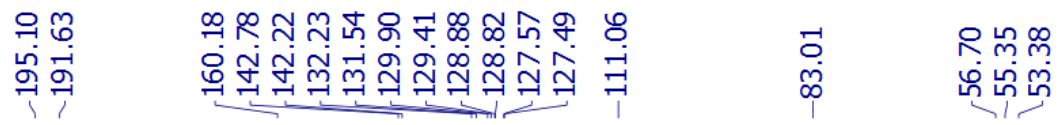

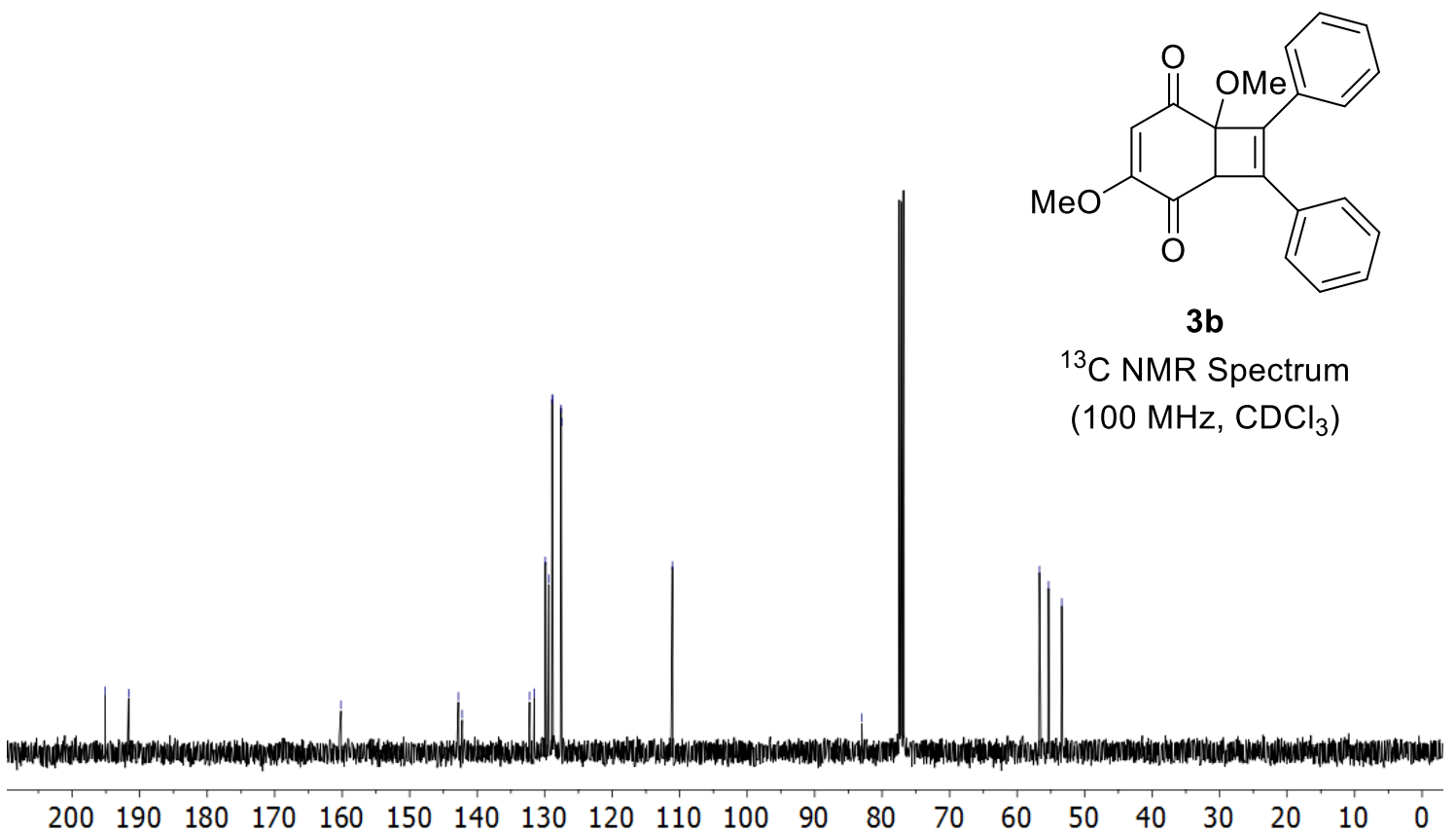

Figure S11. $\mathbf{3 b}{ }^{13} \mathrm{C}-\mathrm{NMR}\left(\mathrm{CDCl}_{3}\right)$ spectra.

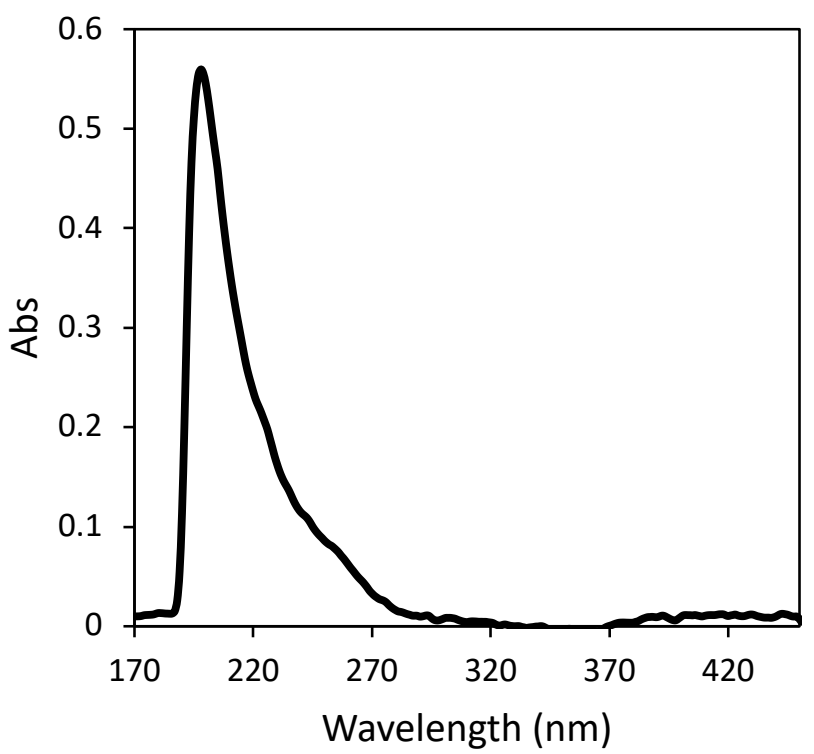

Figure S12. UV/Vis spectra of $3 \mathbf{b}\left[1.0 \cdot 10^{-5} \mathrm{M}\right]$ in acetonitrile. 


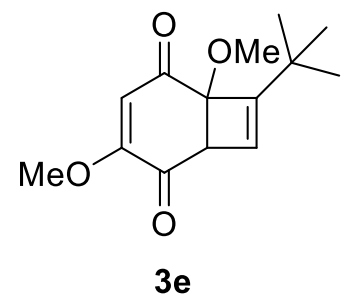

Following the general procedure, but irradiating the reaction mixture for 22 hours, the reaction of $1(100 \mathrm{mg}, 0.595 \mathrm{mmol})$ and alkyne $2 \mathrm{e}(0.74 \mathrm{~mL}, 5.35 \mathrm{mmol})$ rendered $3 e$ as a yellowish oil (41 $\mathrm{mg}, 28 \%)$ after purification by flash chromatography $\left(\mathrm{SiO}_{2}\right.$; Hexane:AcOEt (7:3)).

3e: ${ }^{1} \mathrm{H}-\mathrm{NMR}\left(400 \mathrm{MHz}, \mathrm{CDCl}_{3}\right): \delta=6.21(\mathrm{~d}, \mathrm{~J}=1.4 \mathrm{~Hz}, 1 \mathrm{H}), 5.99(\mathrm{~s}, 1 \mathrm{H}), 3.80(\mathrm{~s}, 3 \mathrm{H}), 3.65(\mathrm{~d}, J=1.4$ $\mathrm{Hz}, 1 \mathrm{H}), 3,29$ (s, 3H), 1.07 (s, 9H). ${ }^{13} \mathrm{C}-\mathrm{NMR}\left(100 \mathrm{MHz}, \mathrm{CDCl}_{3}\right): \delta=196.6,192.5,165.0,160.1$, 131.2, 111.2, 85.8, 56.7, 53.8, 53.0, 29.8, 28.0. HRMS (ESI-TOF) $\mathrm{m} / \mathrm{z}$ : $[\mathrm{M}+\mathrm{H}]^{+}$Calcd. for $\mathrm{C}_{14} \mathrm{H}_{19} \mathrm{O}_{4}$ 251.1278; Found 251.1270. [M+Na] ${ }^{+}$Calcd. for $\mathrm{C}_{14} \mathrm{H}_{18} \mathrm{NaO}_{4}$ 273.1097; Found 273.1088. FT-IR (ATR) v 3434, 3402, 3388, 3317, 3056, 2959, 2925, 2854, 1736, 1729, 1703, 1666, 1602, 1573, $1461,1392,1362,1300,1260,1206,1181,1166,1119,1098,1015,992,965,913,853,837,802$, $765,750,704,651 \mathrm{~cm}^{-1}$.

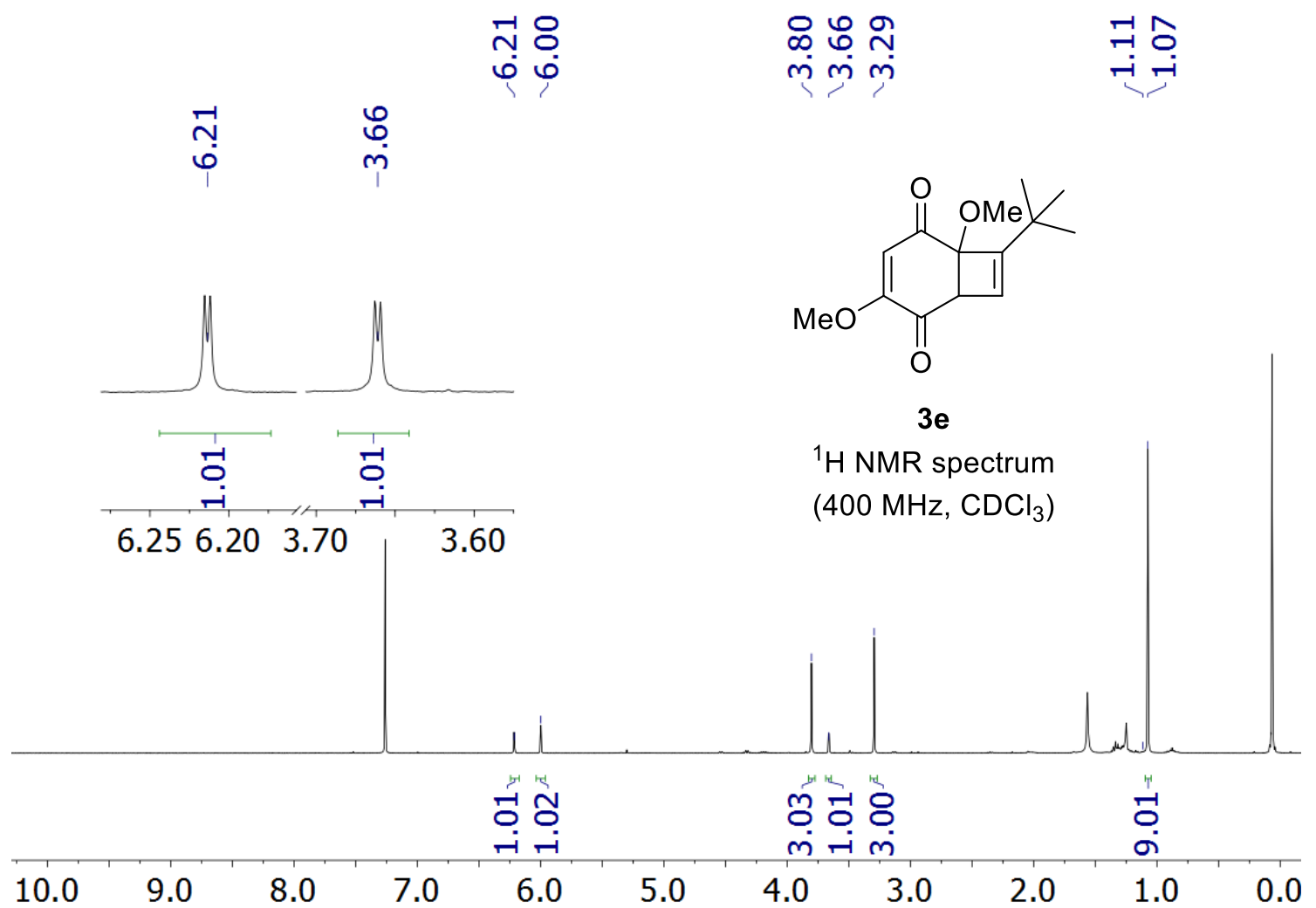

Figure S13. $3 e^{1} \mathrm{H}-\mathrm{NMR}\left(\mathrm{CDCl}_{3}\right)$ spectra. 


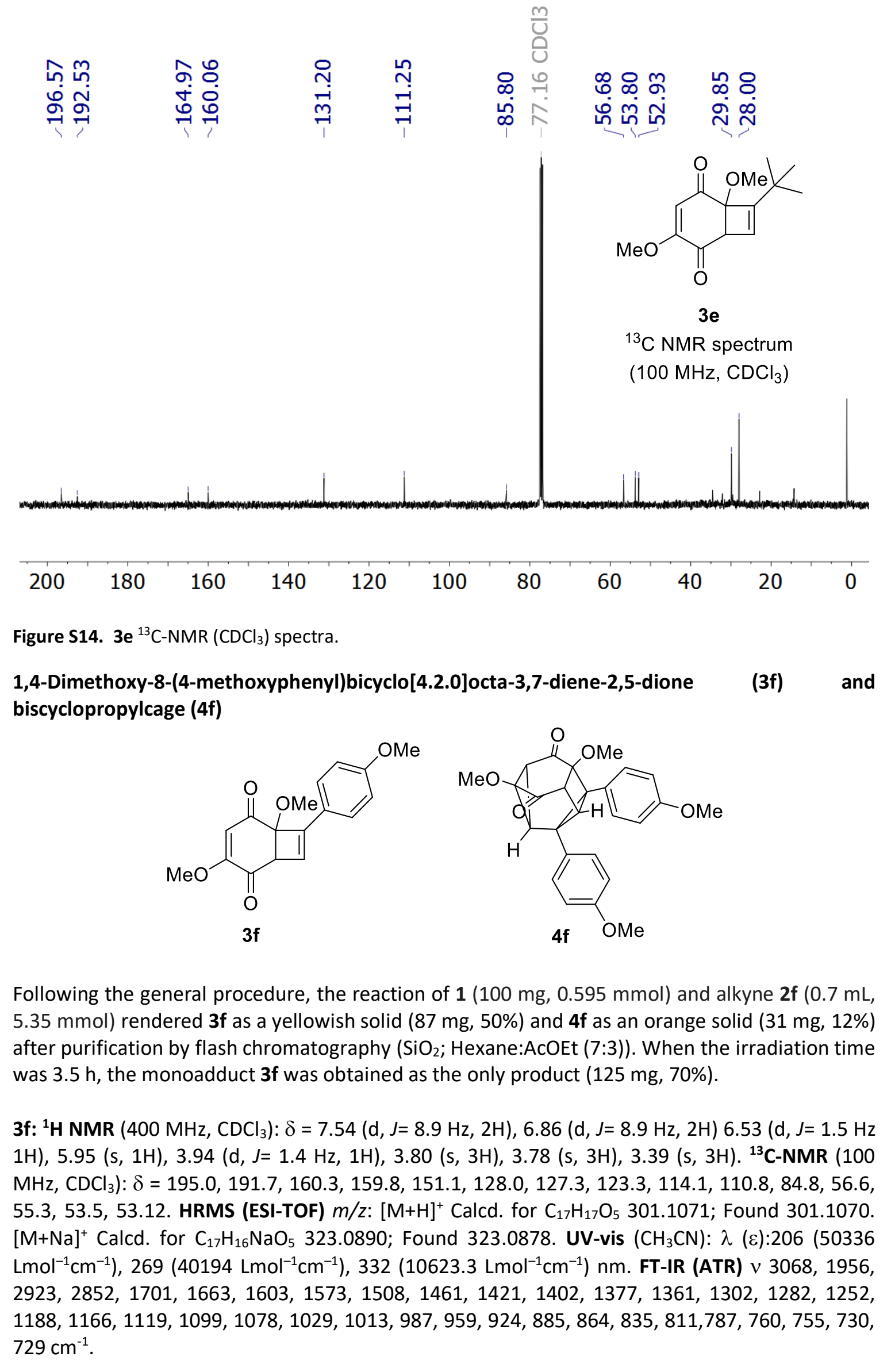



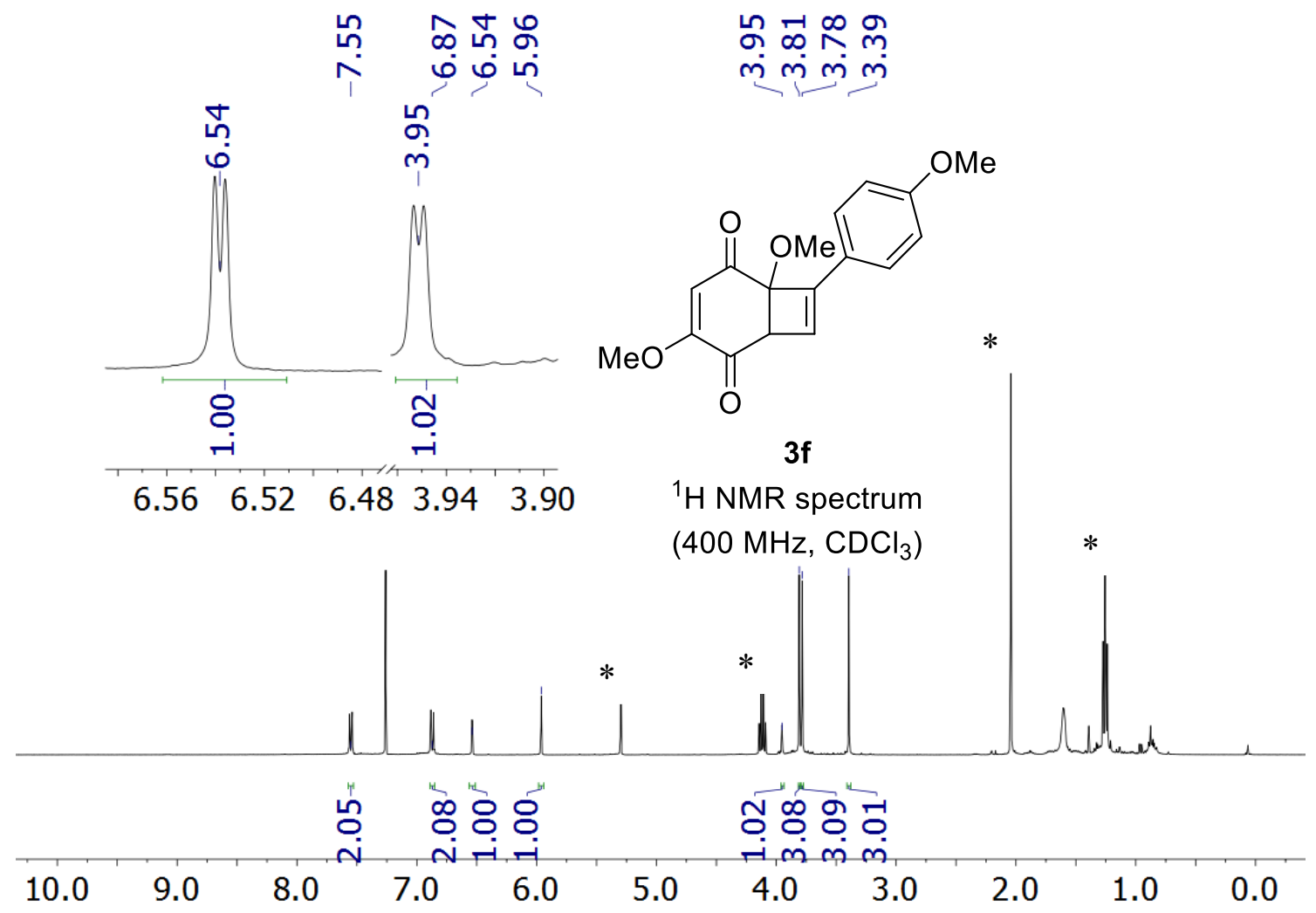

Figure S15. $3 \mathrm{f}^{1} \mathrm{H}-\mathrm{NMR}\left(\mathrm{CDCl}_{3}\right)$ spectra. * Show solvents signal.

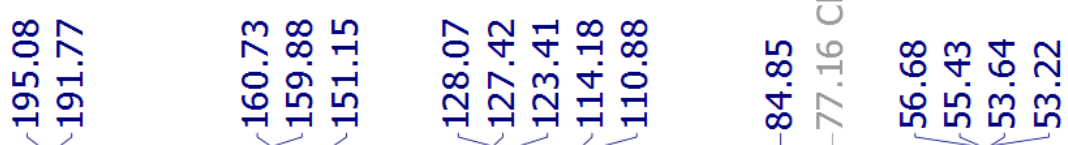

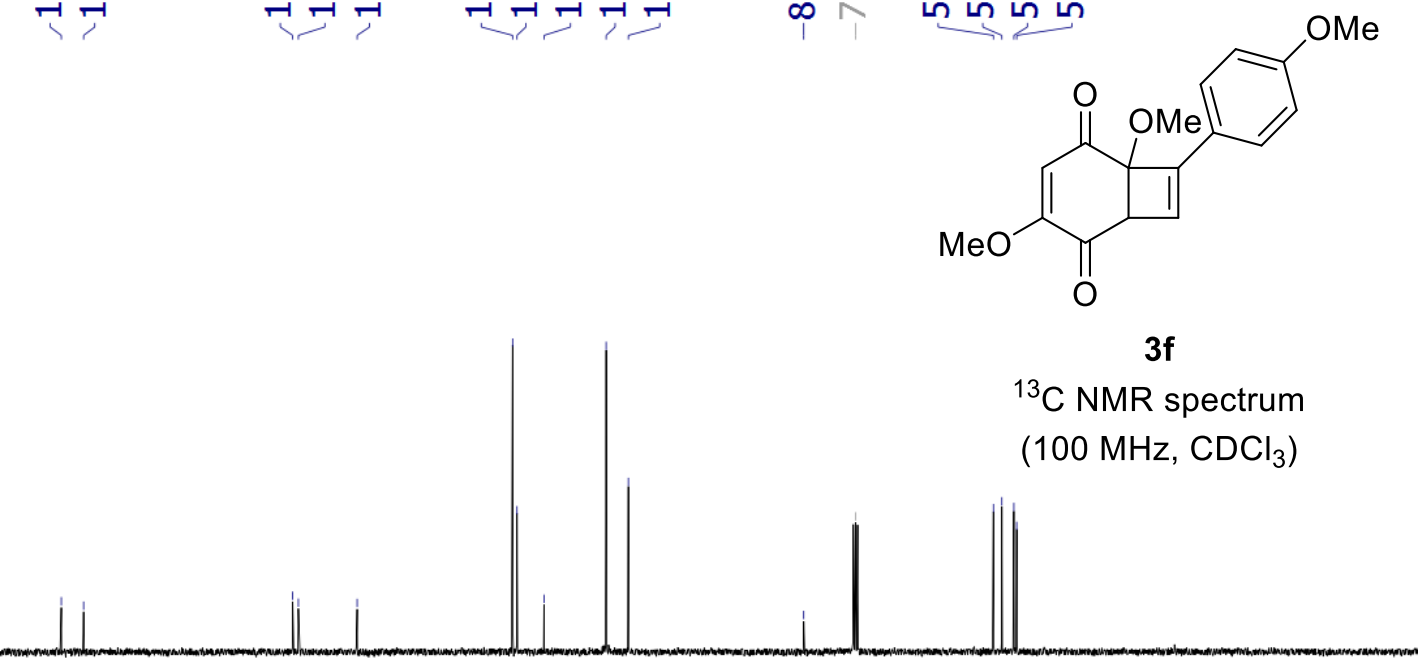

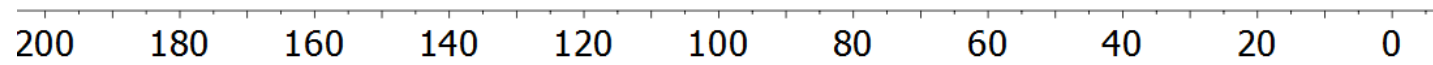

Figure S16. $3 f{ }^{13} \mathrm{C}-\mathrm{NMR}\left(\mathrm{CDCl}_{3}\right)$ spectra. 


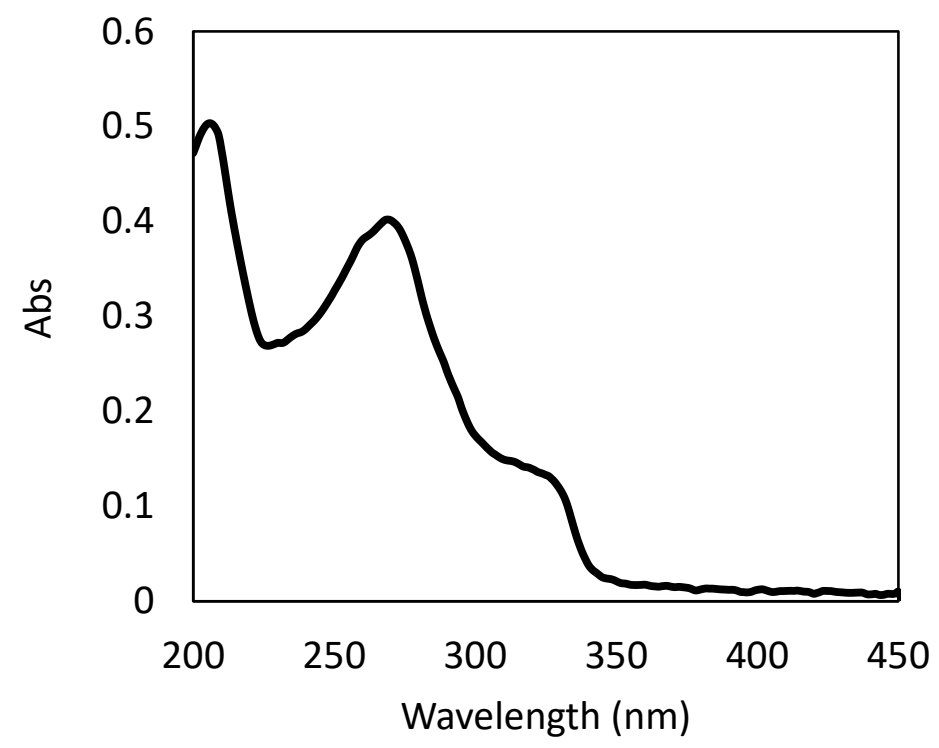

Figure S17. UV/Vis spectra of $\mathbf{3 f}\left[1.0 \cdot 10^{-5} \mathrm{M}\right]$ in acetonitrile.

4f: ${ }^{1} \mathrm{H}-\mathrm{NMR}\left(400 \mathrm{MHz}, \mathrm{CDCl}_{3}\right): \delta=6.91(\mathrm{~d}, J=8.7 \mathrm{~Hz}, 2 \mathrm{H}), 6.64(\mathrm{~m}, 4 \mathrm{H}), 6.56(\mathrm{~d}, J=8.7 \mathrm{~Hz}, 2 \mathrm{H})$, $3.69(\mathrm{~s}, 3 \mathrm{H}), 3.68(\mathrm{~s}, 3 \mathrm{H}), 3.60(\mathrm{~d}, J=4.9 \mathrm{~Hz}, 1 \mathrm{H}), 3.50(\mathrm{~s}, 3 \mathrm{H}), 3.27(\mathrm{~d}, J=4.9 \mathrm{~Hz}, 1 \mathrm{H}), 3.19$ (d, J= $9.8 \mathrm{~Hz}, 1 \mathrm{H}), 2.82$ (d, J= $9.8 \mathrm{~Hz}, 1 \mathrm{H}) .{ }^{13} \mathrm{C}-\mathrm{NMR}\left(100 \mathrm{MHz}, \mathrm{CDCl}_{3}\right): \delta=202.4,200.6,158.8,158.5$, $130.8,128.9,123.8,114.1,113.5,82.6,74.1,57.9,55.3,55.2,53.0,52.7,50.7,45.0,44.6,37.0$, 36.4. HRMS (ESI-TOF) $\mathrm{m} / \mathrm{z}:[\mathrm{M}+\mathrm{H}]^{+}$Calcd. for $\mathrm{C}_{26} \mathrm{H}_{25} \mathrm{O}_{6}$ 433.1646; Found 433.1649. UV-vis $\left(\mathrm{CH}_{3} \mathrm{CN}\right): \lambda(\varepsilon): 231\left(22728 \mathrm{Lmol}^{-1} \mathrm{~cm}^{-1}\right), 264\left(3035 \mathrm{Lmol}^{-1} \mathrm{~cm}^{-1}\right), 345\left(476 \mathrm{Lmol}^{-1} \mathrm{~cm}^{-1}\right) \mathrm{nm}$. FT-IR (ATR) $\vee$ 3648, 3627, 3104, 3042, 2996, 2959, 1932, 1837, 1707, 1611, 1580, 1517, 1463, 1445, $1416,1379,1337,1298,1279,1250,1205,1175,1130,1088,1029,984,926,915,892,830,788$, $741,722,669,582,562 \mathrm{~cm}^{-1}$.

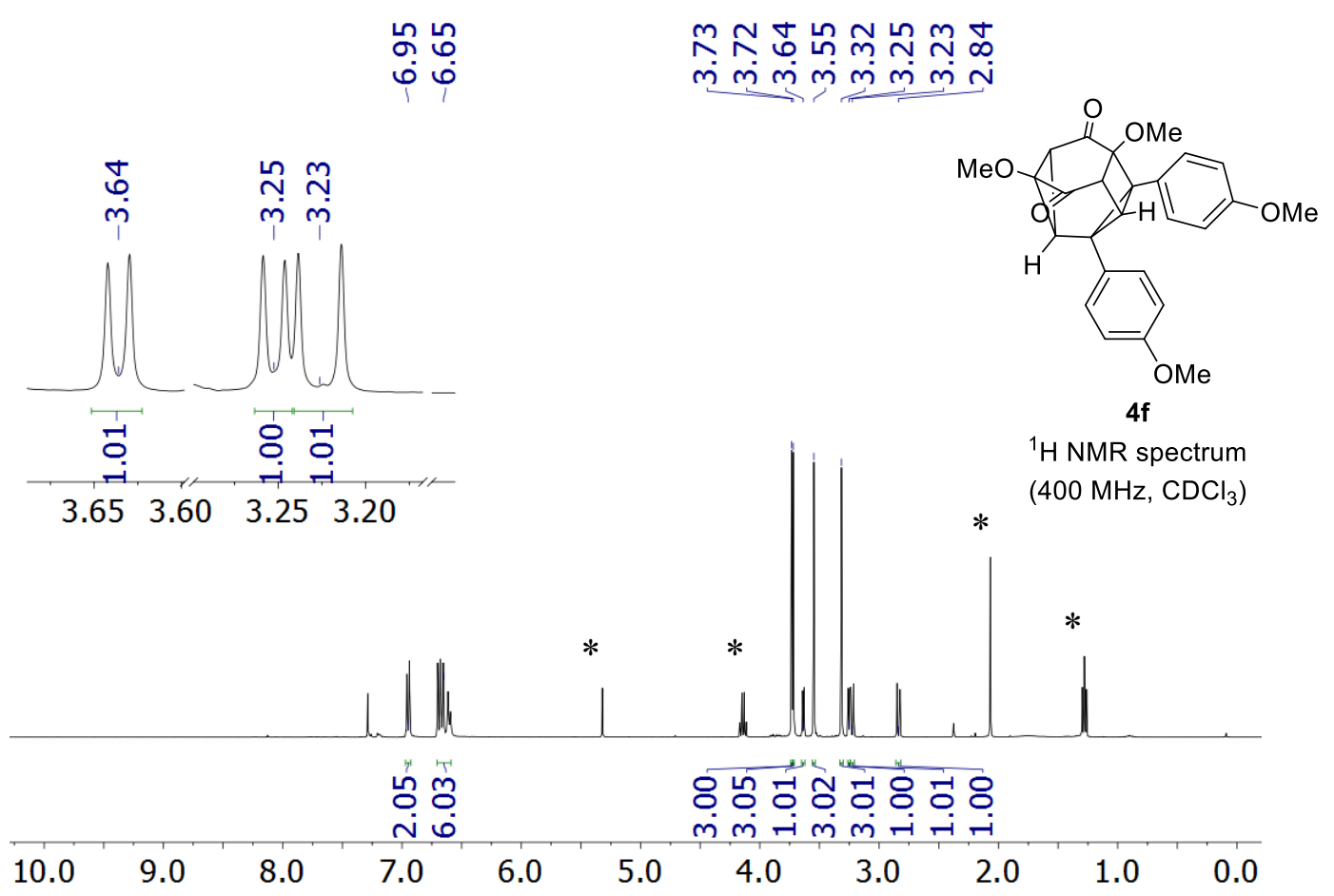

Figure S18. $4 \mathrm{f}^{1} \mathrm{H}-\mathrm{NMR}\left(\mathrm{CDCl}_{3}\right)$ spectra. * Show solvents signal. 

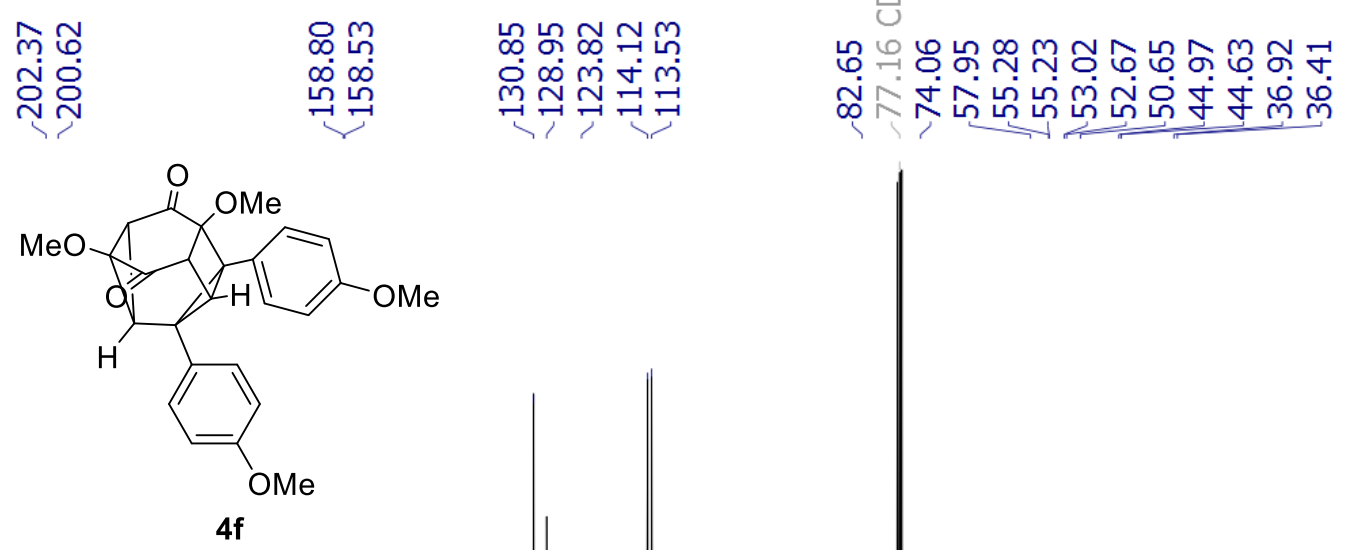

${ }^{13} \mathrm{C}$ NMR spectrum $\left(100 \mathrm{MHz}, \mathrm{CDCl}_{3}\right)$

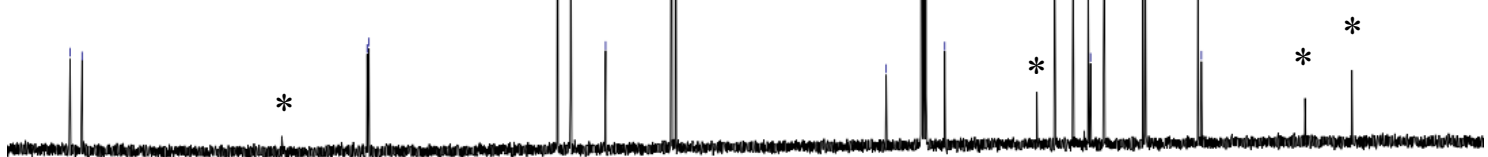

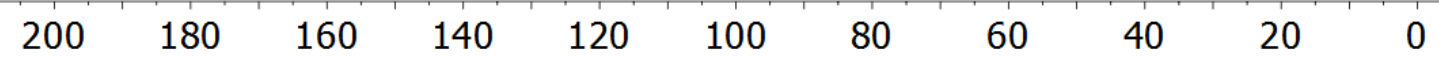

Figure S19. $4 \mathrm{f}^{13} \mathrm{C}-\mathrm{NMR}\left(\mathrm{CDCl}_{3}\right)$ spectra. * Show solvents signal.

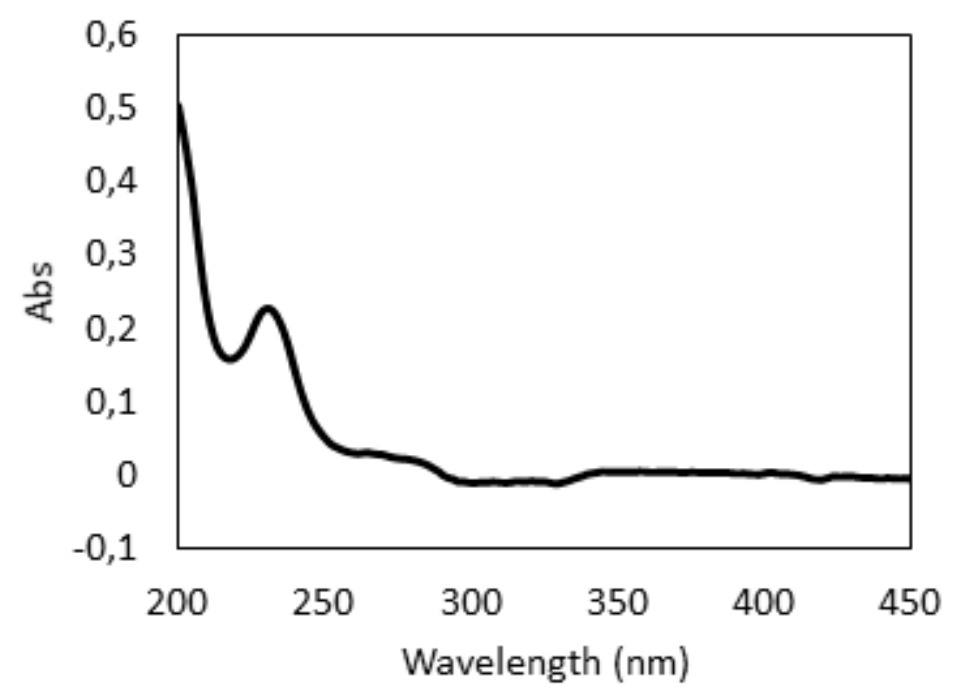

Figure S20. UV/Vis spectra of $\mathbf{4 f}\left[1.0 \cdot 10^{-5} \mathrm{M}\right]$ in acetonitrile. 
<smiles>COC1=CC(=O)C2(OC)C(c3ccc([N+](=O)[O-])cc3)=CC2C1=O</smiles>

$3 g$

Following the general procedure, the reaction of $1(100 \mathrm{mg}, 0.595 \mathrm{mmol})$ and alkyne $\mathbf{2 g}(0.79 \mathrm{~g}$, $5.35 \mathrm{mmol})$ rendered $\mathbf{3 g}$ as a yellowish oil $(29 \mathrm{mg}, 15 \%)$ after purification by flash chromatography $\left(\mathrm{SiO}_{2} ;\right.$ Hexane:AcOEt (6:4)).

3g: ${ }^{1} \mathrm{H}-\mathrm{NMR}\left(400 \mathrm{MHz}, \mathrm{CDCl}_{3}\right): \delta=8.22(\mathrm{~d}, J=8.9 \mathrm{~Hz}, 2 \mathrm{H}), 7.80(\mathrm{~d}, J=8.9 \mathrm{~Hz}, 2 \mathrm{H}) 6.93(\mathrm{~d}, J=1.9 \mathrm{~Hz}$ $1 \mathrm{H}), 6.03(\mathrm{~s}, 1 \mathrm{H}), 4.01(\mathrm{~d}, J=1.9 \mathrm{~Hz}, 1 \mathrm{H}), 3.82(\mathrm{~s}, 3 \mathrm{H}), 3.40(\mathrm{~s}, 3 \mathrm{H}) .{ }^{13} \mathrm{C}-\mathrm{NMR}\left(100 \mathrm{MHz}, \mathrm{CDCl}_{3}\right): \delta=$ 194.4, 190.5, 160.3, 149.5, 148.1, 135.9, 134.8, 127.5, 124.1, 111.4, 84.6, 56.9, 54.3, 53.7. HRMS (ESI-TOF) $\mathrm{m} / \mathrm{z}$ : $[\mathrm{M}+\mathrm{H}]^{+}$Calcd. for $\mathrm{C}_{16} \mathrm{H}_{14} \mathrm{NO}_{6}$ 316.0816; Found 316.0815. [M+Na] $]^{+}$Calcd. for $\mathrm{C}_{16} \mathrm{H}_{13} \mathrm{NNaO}_{6}$ 338.0635; Found 338.0630. UV-vis $\left(\mathrm{CH}_{3} \mathrm{CN}\right): \lambda(\varepsilon): 225\left(5420 \mathrm{Lmol}^{-1} \mathrm{~cm}^{-1}\right), 280$ (8882 $\left.\mathrm{Lmol}^{-1} \mathrm{~cm}^{-1}\right), 349\left(2088 \mathrm{Lmol}^{-1} \mathrm{~cm}^{-1}\right) \mathrm{nm}$. FT-IR (ATR) $\vee 3639,3391,3348,3307,3100,3073$, 2957, 2925, 1852, 2627, 2447, 2256, 1798, 1703, 1661, 1601, 1517, 1489, 1458, 1412, 1343, $1319,1285,1255,1240,1186,1166,1107,1079,1037,1011,987,960,913,850,814,762,742$, $699,646,542 \mathrm{~cm}^{-1}$.

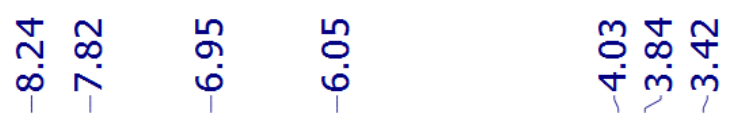

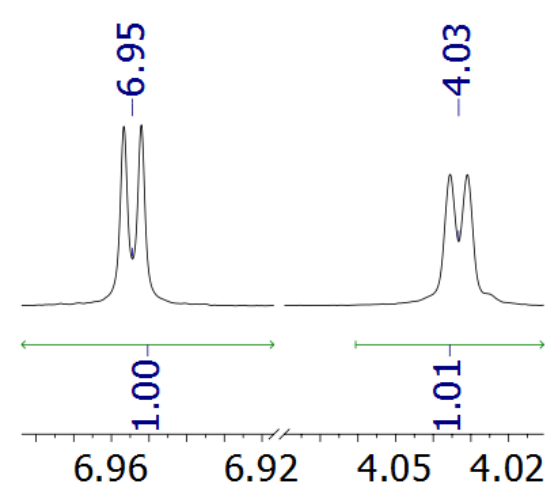<smiles>COC1=CC(=O)C2(OC)C(c3ccc([N+](=O)[O-])cc3)=CC2C1=O</smiles>

$3 g$

${ }^{1} \mathrm{H}$ NMR spectrum $\left(400 \mathrm{MHz}, \mathrm{CDCl}_{3}\right.$ )

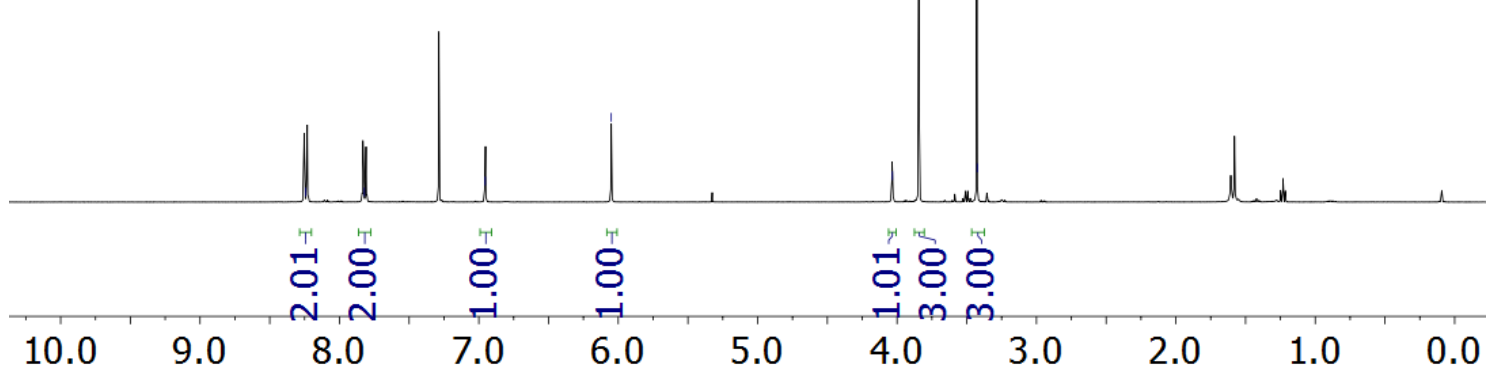

Figure S21. 3g ${ }^{1} \mathrm{H}-\mathrm{NMR}\left(\mathrm{CDCl}_{3}\right)$ spectra. 


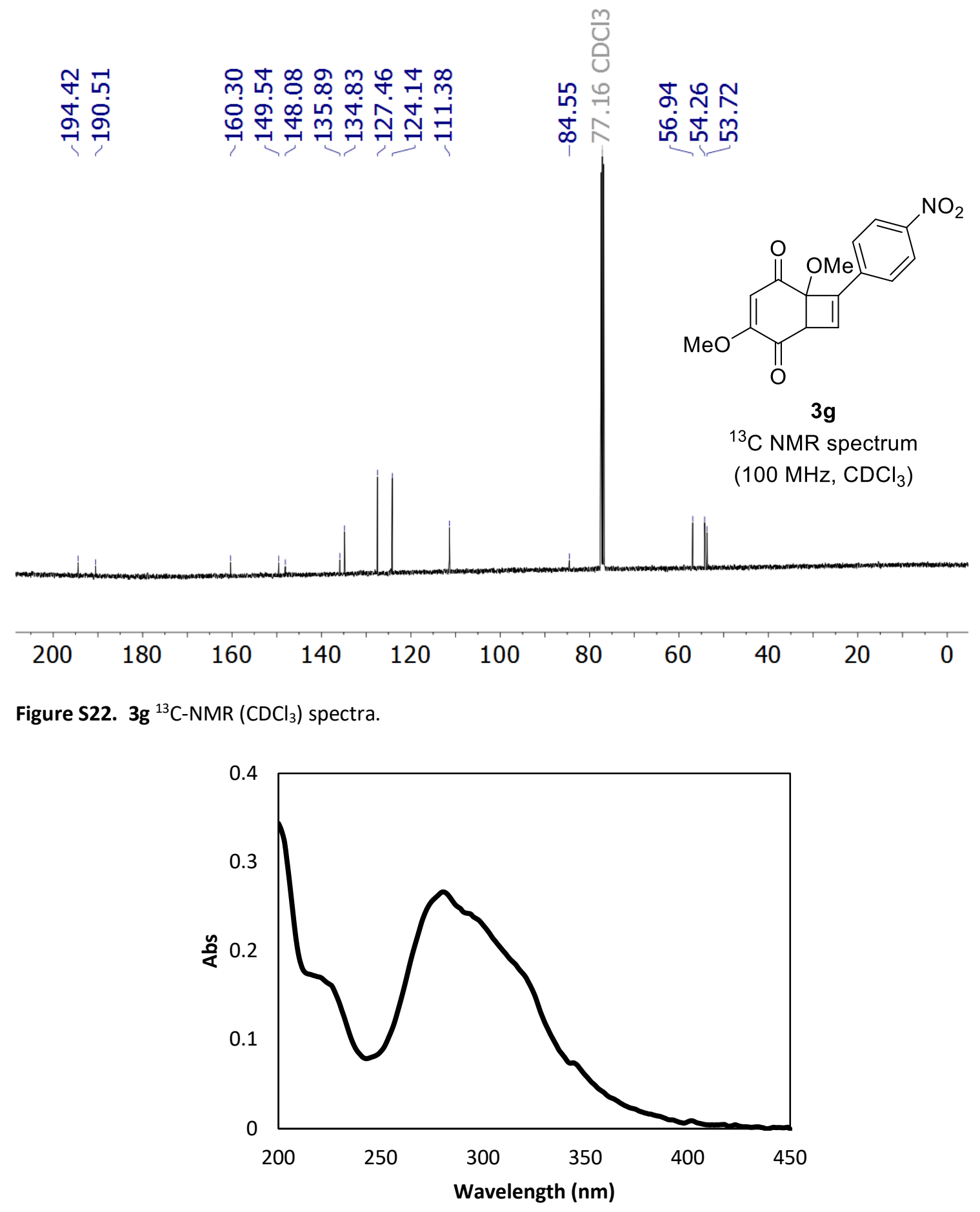

Figure S23. UV/Vis spectra of $\mathbf{3 g}\left[3.0 \cdot 10^{-5} \mathrm{M}\right]$ in acetonitrile. 


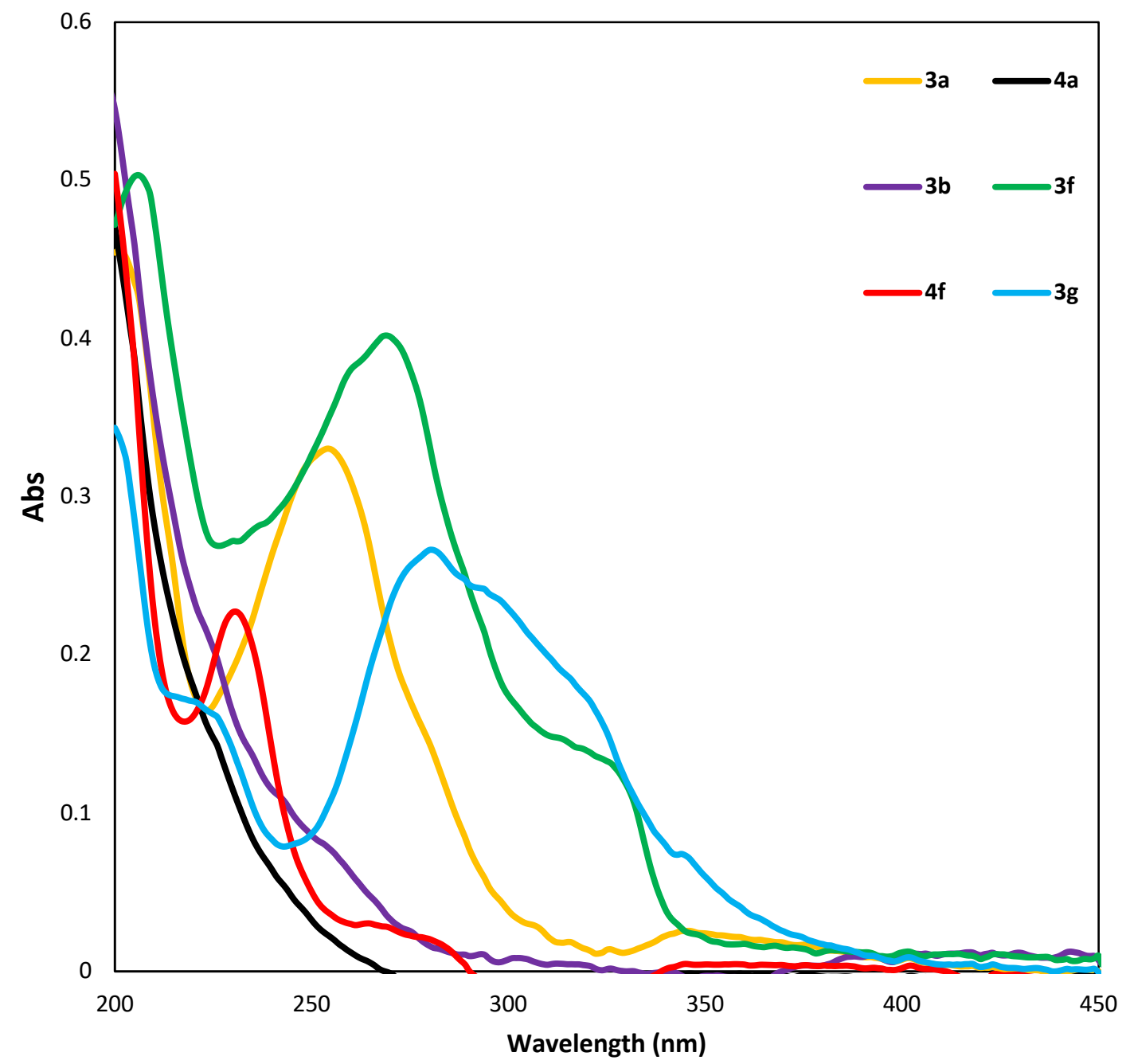

Figure S24. UV/Vis spectra of 3a (yellow) $\left[1.0 \cdot 10^{-5} \mathrm{M}\right], \mathbf{4 a}$ (black) $\left[1.0 \cdot 10^{-5} \mathrm{M}\right], \mathbf{3 b}$ (purple) $\left[1.0 \cdot 10^{-5} \mathrm{M}\right], \mathbf{3 f}$ (green) $\left[1.0 \cdot 10^{-5} \mathrm{M}\right], \mathbf{4 f}(\mathrm{red})\left[1.0 \cdot 10^{-5} \mathrm{M}\right]$ and $3 \mathrm{~g}$ (blue) $\left[3.0 \cdot 10^{-5} \mathrm{M}\right]$ in acetonitrile. 


\section{Detailed computational methods}

The geometries of all the molecules were fully optimized by using the M06 functional ${ }^{9}$ with the double- $\zeta$ quality basis set $6-31+\mathrm{G}(\mathrm{d}, \mathrm{p})$. This basis set has been used within some of the training sets to parametrize the MO6 functional and it is known to provide superior performance relative to its size. This basis set contains polarization and diffuse functions which are expected to provide enough flexibility to describe low energy excited states. The effect of the solvent (Toluene) was taken into account using the Polarizable Continuum Model (PCM) ${ }^{10}$ with the default parameters implemented in the Gaussian 09 package. ${ }^{11}$ Harmonic analysis was performed to confirm the nature of minima and transition states. The wave function stability was confirmed in all stationary points. ${ }^{12}$ Excited states were computed via the time-dependent formulation of the Kohn-Sham equations. These calculations were run with the Gaussian 16 program, which currently implements analytical first and second derivatives for excited states computed within the TDDFT framework, hence allowing for geometry optimization and full characterization of stationary points. This methodology however has some caveats that are worth mentioning. TDDFT is based on linear response theory from a single determinant reference. In those cases where the ground state is not correctly described with a single determinant (this can usually happen in the vicinity of transition states or in diradicaloid states) the linear response is also prone to fail. Similarly, even when the ground state is reasonably described with a single determinant, the excited state can also show near degeneracy issues, and lead to incorrect excitation energies. These two situations can be evaluated via stability checks. ${ }^{12}$ This is relatively straight forward for the ground state, but it is not implemented for excited states. Often times, near degeneracy in the excited state during optimization procedures result in energy hopping and erratic convergence. These symptoms are easy to spot for the trained eye and, in the current study, we have not observed them. Finally, despite some algorithms implemented to avoid it, geometry optimization withing TDDFT can lead to accidental surface hops due to nearby states. The chance for these events has been minimized via a small stepsize and generous use of IRC calculations to obtain educated guesses for each stationary point along the alternate mechanisms. It is also worth mentioning that in the photophysical events (the vertical absorption, for instance) only the fast components of the polarizability are allowed to relax within the continuum solvation model. On the contrary, for those events that imply nuclear motion (excited state optimizations, IRCs, etc) both fast and slow components are allowed to relax. 


\section{Reaction profile with exo approach}

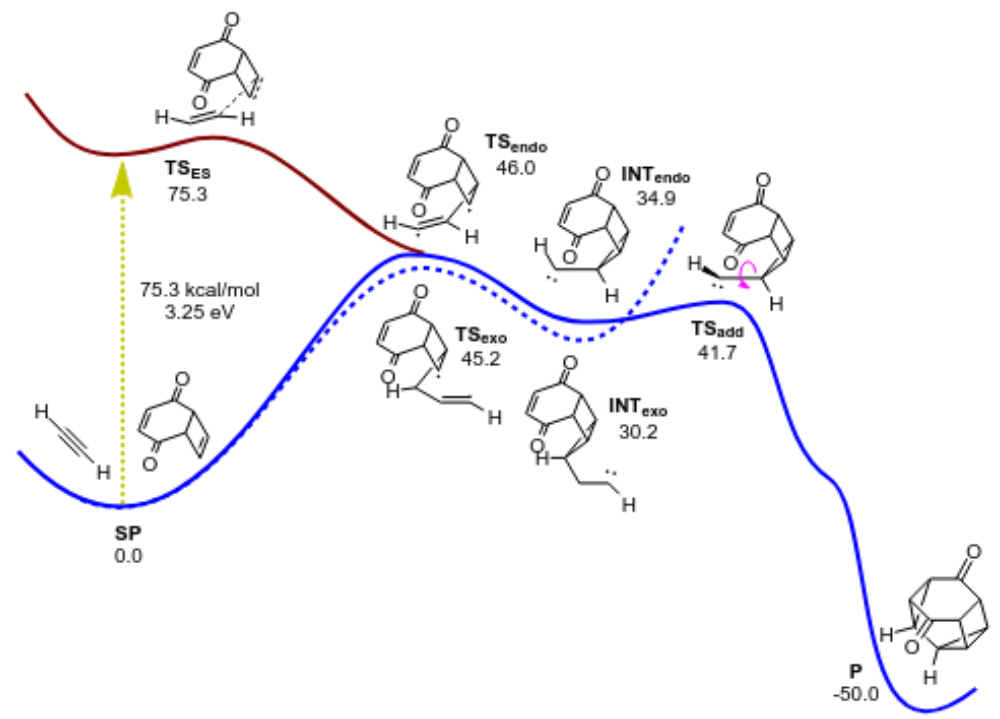

Figure S1: Reaction profile showing the ease of reversibility when the initial orientation is exo. 


\section{Cartesian Coordinates}

\subsection{Reaction paths on the article}

Int1-S0

SCF Energy: -535.790757115

ZPE-corrected Energy: -535.641074

$\Delta \mathrm{U}:-535.631187$

$\Delta \mathrm{H}:-535.630243$

$\Delta \mathrm{G}:-535.676473$

Num. Imaginary Frequencies: 0

$\begin{array}{rrrr}\mathrm{C} & -2.029295 & -0.675947 & 0.129941 \\ \mathrm{C} & 1.145905 & 0.001732 & 1.946711 \\ \mathrm{C} & -2.031823 & 0.668360 & 0.130012 \\ \mathrm{C} & -0.804832 & 1.460973 & -0.143194 \\ \mathrm{C} & 0.246562 & 0.772539 & -0.968514 \\ \mathrm{C} & 0.249412 & -0.771320 & -0.968718 \\ \mathrm{C} & -0.799316 & -1.463833 & -0.143392 \\ \mathrm{C} & 1.714027 & -0.734673 & -0.549041 \\ \mathrm{C} & 1.977104 & 0.003388 & 0.806866 \\ \mathrm{C} & 1.711299 & 0.741133 & -0.548747 \\ \mathrm{O} & -0.676133 & -2.609017 & 0.251886 \\ \mathrm{O} & -0.686131 & 2.606627 & 0.252092 \\ \mathrm{H} & -2.904138 & -1.248043 & 0.433843 \\ \mathrm{H} & -2.908813 & 1.237130 & 0.433983 \\ \mathrm{H} & 0.160964 & 1.176369 & -1.986090 \\ \mathrm{H} & 0.165276 & -1.175152 & -1.986414 \\ \mathrm{H} & 2.487465 & -1.435461 & -0.846106 \\ \mathrm{H} & 0.083481 & 0.000041 & 1.638840 \\ \mathrm{H} & 3.057429 & 0.005331 & 0.974224 \\ \mathrm{H} & 2.482187 & 1.444791 & -0.845647\end{array}$


Int2-S0

SCF Energy: -535.801371465

ZPE-corrected Energy: -535.651432

$\Delta \mathrm{U}:-535.641748$

$\Delta \mathrm{H}:-535.640804$

$\Delta \mathrm{G}:-535.686235$

Num. Imaginary Frequencies: 0

$\begin{array}{rrrr}\mathrm{C} & -2.016448 & -0.680972 & 0.098565 \\ \mathrm{C} & 0.826256 & 0.003059 & 1.727289 \\ \mathrm{C} & -2.023577 & 0.660080 & 0.098641 \\ \mathrm{C} & -0.785193 & 1.447561 & -0.132784 \\ \mathrm{C} & 0.266978 & 0.773861 & -0.970280 \\ \mathrm{C} & 0.275039 & -0.770468 & -0.970669 \\ \mathrm{C} & -0.769860 & -1.455367 & -0.133147 \\ \mathrm{C} & 1.734263 & -0.724090 & -0.534805 \\ \mathrm{C} & 1.911787 & 0.009756 & 0.840575 \\ \mathrm{C} & 1.726550 & 0.742527 & -0.534264 \\ \mathrm{O} & -0.650952 & -2.607917 & 0.248080 \\ \mathrm{O} & -0.678289 & 2.601122 & 0.248916 \\ \mathrm{H} & -2.897758 & -1.261736 & 0.364871 \\ \mathrm{H} & -2.910989 & 1.231432 & 0.365032 \\ \mathrm{H} & 0.182918 & 1.182971 & -1.986040 \\ \mathrm{H} & 0.195018 & -1.180002 & -1.986584 \\ \mathrm{H} & 2.521379 & -1.429257 & -0.781000 \\ \mathrm{H} & 1.192511 & 0.003997 & 2.770627 \\ \mathrm{H} & 2.969839 & 0.015253 & 1.122452 \\ \mathrm{H} & 2.506248 & 1.456031 & -0.780047\end{array}$


Monoadduct

SCF Energy: -458.561748613

ZPE-corrected Energy: -458.443056

$\triangle \mathrm{U}:-458.435112$

$\Delta \mathrm{H}:-458.434168$

$\Delta \mathrm{G}:-458.476405$

Num. Imaginary Frequencies: 0

$\begin{array}{lrrr}\mathrm{C} & -0.670957 & -1.588985 & -0.373890 \\ \mathrm{C} & 0.671367 & -1.588768 & -0.374130 \\ \mathrm{C} & 1.485202 & -0.469254 & 0.146601 \\ \mathrm{C} & 0.778956 & 0.791527 & 0.563262 \\ \mathrm{C} & -0.779090 & 0.791407 & 0.563536 \\ \mathrm{C} & -1.485035 & -0.469528 & 0.146682 \\ \mathrm{C} & -0.669283 & 1.830703 & -0.539598 \\ \mathrm{C} & 0.668644 & 1.830662 & -0.539951 \\ \mathrm{O} & -2.701168 & -0.559265 & 0.196763 \\ \mathrm{O} & 2.701347 & -0.558733 & 0.196995 \\ \mathrm{H} & -1.237739 & -2.438049 & -0.752198 \\ \mathrm{H} & 1.238304 & -2.437662 & -0.752591 \\ \mathrm{H} & 1.237522 & 1.159705 & 1.489509 \\ \mathrm{H} & -1.237494 & 1.159083 & 1.490066 \\ \mathrm{H} & -1.436425 & 2.367249 & -1.089571 \\ \mathrm{H} & 1.435576 & 2.367077 & -1.090351\end{array}$


$\mathrm{PF}$

SCF Energy: -535.935774273

ZPE-corrected Energy: -535.779069

$\Delta \mathrm{U}:-535.771296$

$\Delta \mathrm{H}:-535.770351$

$\Delta \mathrm{G}:-535.811878$

Num. Imaginary Frequencies: 0

$\begin{array}{lrrr}\mathrm{C} & -0.784357 & 0.484575 & -1.039525 \\ \mathrm{C} & 0.784689 & 0.486740 & -1.038873 \\ \mathrm{C} & 1.434037 & -0.674266 & -0.336640 \\ \mathrm{C} & 0.771018 & -1.052823 & 0.932264 \\ \mathrm{C} & -0.768327 & -1.055546 & 0.931198 \\ \mathrm{C} & -1.431986 & -0.677389 & -0.337221 \\ \mathrm{C} & -0.758086 & 1.754446 & -0.173685 \\ \mathrm{C} & 0.754118 & 1.756267 & -0.173125 \\ \mathrm{O} & 2.437403 & -1.232112 & -0.746391 \\ \mathrm{O} & -2.435677 & -1.235190 & -0.746184 \\ \mathrm{H} & 1.260914 & -1.830321 & 1.512083 \\ \mathrm{C} & -0.000436 & 0.025526 & 1.643962 \\ \mathrm{H} & -1.256174 & -1.834298 & 1.511035 \\ \mathrm{H} & -1.212062 & 0.621550 & -2.034820 \\ \mathrm{H} & 1.212956 & 0.624707 & -2.033814 \\ \mathrm{H} & 1.450248 & 2.585962 & -0.242831 \\ \mathrm{H} & -0.001345 & -0.046275 & 2.729038 \\ \mathrm{C} & -0.002264 & 1.422768 & 1.078408 \\ \mathrm{H} & -0.002945 & 2.212719 & 1.825319 \\ \mathrm{H} & -1.455830 & 2.582592 & -0.245989\end{array}$


PP1

SCF Energy: -458.561748625

ZPE-corrected Energy: -458.443057

$\Delta \mathrm{U}:-458.435112$

$\Delta \mathrm{H}:-458.434168$

$\Delta \mathrm{G}:-458.476407$

Num. Imaginary Frequencies: 0

$\begin{array}{rrrr}\mathrm{C} & 0.670899 & -1.589149 & 0.373704 \\ \mathrm{C} & -0.671413 & -1.589005 & 0.373712 \\ \mathrm{C} & -1.485218 & -0.469210 & -0.146519 \\ \mathrm{C} & -0.778979 & 0.791567 & -0.563259 \\ \mathrm{C} & 0.779109 & 0.791339 & -0.563526 \\ \mathrm{C} & 1.484995 & -0.469578 & -0.146567 \\ \mathrm{C} & 0.669381 & 1.830785 & 0.539450 \\ \mathrm{C} & -0.668546 & 1.830898 & 0.539767 \\ \mathrm{O} & 2.701143 & -0.559254 & -0.196446 \\ \mathrm{O} & -2.701381 & -0.558601 & -0.196549 \\ \mathrm{H} & 1.237695 & -2.438272 & 0.751862 \\ \mathrm{H} & -1.238379 & -2.438015 & 0.751866 \\ \mathrm{H} & -1.237490 & 1.159551 & -1.489622 \\ \mathrm{H} & 1.237519 & 1.158887 & -1.490109 \\ \mathrm{H} & 1.436608 & 2.367306 & 1.089336 \\ \mathrm{H} & -1.435417 & 2.367504 & 1.090066\end{array}$

PP2

SCF Energy: -77.2716615980

ZPE-corrected Energy: -77.246509

$\Delta \mathrm{U}:-77.243323$

$\Delta \mathrm{H}:-77.242378$

$\Delta \mathrm{G}:-77.263860$

Num. Imaginary Frequencies: 0

$\begin{array}{llll}\text { C } & 0.001564 & 0.603814 & 0.000000\end{array}$

$\begin{array}{llll}\text { C } & 0.001564 & -0.603808 & -0.000000\end{array}$

$\mathrm{H} \quad-0.009260 \quad-1.672109-0.000000$

$\begin{array}{llll}\mathrm{H} & -0.009508 & 1.672071 & 0.000000\end{array}$ 


\begin{tabular}{|c|c|c|c|}
\hline \\
\hline \multicolumn{4}{|c|}{$\begin{array}{l}\text { SP } \\
\text { SCF Energy: -535.837797001 }\end{array}$} \\
\hline \multicolumn{4}{|c|}{ ZPE-corrected Energy: -535.691284 } \\
\hline \multicolumn{4}{|c|}{$\Delta \mathrm{U}:-535.678968$} \\
\hline \multicolumn{4}{|c|}{$\Delta \mathrm{H}:-535.678024$} \\
\hline \multicolumn{4}{|c|}{$\Delta \mathrm{G}:-535.732107$} \\
\hline \multicolumn{4}{|c|}{ Num. Imaginary Frequencies: 0} \\
\hline $\mathrm{C}$ & -0.856496 & 1.645667 & -0.041847 \\
\hline $\mathrm{C}$ & 3.174053 & 0.323346 & 0.900629 \\
\hline $\mathrm{C}$ & 0.305515 & 1.403048 & -0.668421 \\
\hline $\mathrm{C}$ & 0.666750 & 0.082626 & -1.226570 \\
\hline $\mathrm{C}$ & -0.226993 & -1.086586 & -0.920803 \\
\hline $\mathrm{C}$ & -1.579731 & -0.805675 & -0.199851 \\
\hline $\mathrm{C}$ & -1.897229 & 0.617614 & 0.169479 \\
\hline $\mathrm{C}$ & -1.009732 & -1.621468 & 0.948492 \\
\hline $\mathrm{C}$ & 2.563596 & 0.428237 & 1.938003 \\
\hline $\mathrm{C}$ & 0.155242 & -1.854923 & 0.333552 \\
\hline $\mathrm{O}$ & -2.969602 & 0.914709 & 0.672024 \\
\hline $\mathrm{O}$ & 1.683708 & -0.053955 & -1.888762 \\
\hline $\mathrm{H}$ & -1.098554 & 2.636552 & 0.338658 \\
\hline $\mathrm{H}$ & 1.046828 & 2.187252 & -0.813659 \\
\hline $\mathrm{H}$ & -0.310730 & -1.707131 & -1.821754 \\
\hline $\mathrm{H}$ & -2.459705 & -1.247732 & -0.683724 \\
\hline $\mathrm{H}$ & -1.441367 & -1.899899 & 1.905147 \\
\hline $\mathrm{H}$ & 3.692129 & 0.228949 & -0.030409 \\
\hline $\mathrm{H}$ & 2.026290 & 0.520129 & 2.857257 \\
\hline $\mathrm{H}$ & 1.062 & -2.3 & \\
\hline
\end{tabular}


TS1-S1

SCF Energy: -535.765237668

ZPE-corrected Energy: -535.576166

$\Delta \mathrm{U}:-535.565627$

$\Delta \mathrm{H}:-535.564682$

$\Delta \mathrm{G}:-535.612161$

Num. Imaginary Frequencies: 1

Imaginary Frequency: -421.8906

$\begin{array}{rrrr}\mathrm{C} & 0.856189 & -1.672848 & 0.493550 \\ \mathrm{C} & -0.301135 & 0.882177 & 2.082625 \\ \mathrm{C} & -0.538684 & -1.831608 & 0.382027 \\ \mathrm{C} & -1.391467 & -0.940822 & -0.308122 \\ \mathrm{C} & -0.707656 & 0.151355 & -1.120434 \\ \mathrm{C} & 0.847374 & 0.256590 & -1.102905 \\ \mathrm{C} & 1.615064 & -0.646126 & -0.112163 \\ \mathrm{C} & 0.677267 & 1.688189 & -0.764784 \\ \mathrm{C} & -0.540813 & 1.871543 & 1.375403 \\ \mathrm{C} & -0.714330 & 1.588008 & -0.643060 \\ \mathrm{O} & 2.825961 & -0.419216 & 0.046306 \\ \mathrm{O} & -2.637362 & -0.961956 & -0.302328 \\ \mathrm{H} & 1.422149 & -2.354478 & 1.126735 \\ \mathrm{H} & -1.026921 & -2.646087 & 0.913465 \\ \mathrm{H} & -1.123751 & 0.110493 & -2.136089 \\ \mathrm{H} & 1.346078 & 0.123937 & -2.074555 \\ \mathrm{H} & 1.370326 & 2.520997 & -0.678084 \\ \mathrm{H} & -0.074411 & -0.053489 & 2.550494 \\ \mathrm{H} & -0.735359 & 2.930692 & 1.365634 \\ \mathrm{H} & -1.497759 & 2.338559 & -0.712246\end{array}$


TS2-S0

SCF Energy: -535.770610486

ZPE-corrected Energy: -535.623348

$\Delta \mathrm{U}:-535.613329$

$\Delta \mathrm{H}:-535.612385$

$\Delta \mathrm{G}:-535.658748$

Num. Imaginary Frequencies: 1

Imaginary Frequency: -434.1449

$\begin{array}{rrrr}\mathrm{C} & -1.520037 & 1.437047 & 0.274084 \\ \mathrm{C} & 0.961121 & -0.706920 & 1.997146 \\ \mathrm{C} & -0.324828 & 2.042323 & 0.168715 \\ \mathrm{C} & 0.901043 & 1.331954 & -0.279617 \\ \mathrm{C} & 0.691256 & 0.066664 & -1.064186 \\ \mathrm{C} & -0.707795 & -0.593131 & -1.010320 \\ \mathrm{C} & -1.712780 & 0.003614 & -0.050308 \\ \mathrm{C} & -0.003521 & -1.874637 & -0.681803 \\ \mathrm{C} & 1.128208 & -1.604380 & 1.059751 \\ \mathrm{C} & 1.269443 & -1.276079 & -0.609355 \\ \mathrm{O} & -2.651245 & -0.633357 & 0.388369 \\ \mathrm{O} & 2.009531 & 1.795063 & -0.077241 \\ \mathrm{H} & -2.387874 & 1.954418 & 0.679270 \\ \mathrm{H} & -0.179327 & 3.073213 & 0.487207 \\ \mathrm{H} & 1.007605 & 0.276908 & -2.093844 \\ \mathrm{H} & -1.211216 & -0.628767 & -1.989124 \\ \mathrm{H} & -0.356028 & -2.893298 & -0.562591 \\ \mathrm{H} & 0.478395 & 0.262558 & 1.900843 \\ \mathrm{H} & 1.437759 & -2.643119 & 1.147422 \\ \mathrm{H} & 2.251741 & -1.654296 & -0.882855\end{array}$


TS3-S0

SCF Energy: -535.779828246

ZPE-corrected Energy: -535.631159

$\triangle \mathrm{U}:-535.622071$

$\Delta \mathrm{H}:-535.621127$

$\Delta \mathrm{G}:-535.665379$

$S^{2}$ before (0.0199) and after higher multiplicity projection $(0.0000)$

Num. Imaginary Frequencies: 1

Imaginary Frequency: -209.0571

$\begin{array}{lrrr}\mathrm{C} & -0.487903 & 1.821751 & 0.383407 \\ \mathrm{C} & -0.197354 & -0.712557 & 2.046674 \\ \mathrm{C} & 0.851730 & 1.687239 & 0.458645 \\ \mathrm{C} & 1.597654 & 0.616539 & -0.232823 \\ \mathrm{C} & 0.788854 & -0.427406 & -0.950455 \\ \mathrm{C} & -0.751321 & -0.291340 & -1.027430 \\ \mathrm{C} & -1.384791 & 0.880045 & -0.327924 \\ \mathrm{C} & -0.897607 & -1.682982 & -0.381628 \\ \mathrm{C} & -0.276033 & -1.700502 & 0.987744 \\ \mathrm{C} & 0.613891 & -1.774135 & -0.256247 \\ \mathrm{O} & -2.592728 & 1.056817 & -0.334089 \\ \mathrm{O} & 2.816614 & 0.578687 & -0.211573 \\ \mathrm{H} & -0.996281 & 2.638466 & 0.894673 \\ \mathrm{H} & 1.456360 & 2.391844 & 1.026516 \\ \mathrm{H} & 1.234358 & -0.581158 & -1.939575 \\ \mathrm{H} & -1.115922 & -0.296698 & -2.060163 \\ \mathrm{H} & -1.581329 & -2.454883 & -0.723403 \\ \mathrm{H} & -0.998876 & 0.046176 & 1.925475 \\ \mathrm{H} & -0.203396 & -2.700474 & 1.434433 \\ \mathrm{H} & 1.271270 & -2.627217 & -0.392437\end{array}$


Int0-T1

SCF Energy: -535.786051361

ZPE-corrected Energy: -535.638890

$\triangle \mathrm{U}:-535.627985$

$\Delta \mathrm{H}:-535.627041$

$\Delta \mathrm{G}:-535.677489$

$S^{2}$ before (2.0215) and after higher multiplicity projection (2.0002)

Num. Imaginary Frequencies: 0

$\begin{array}{lrrr}\mathrm{C} & 1.725846 & 0.901218 & -0.873865 \\ \mathrm{C} & -3.318313 & -0.788127 & -0.945671 \\ \mathrm{C} & 0.677709 & 1.705519 & -0.632446 \\ \mathrm{C} & -0.335915 & 1.421001 & 0.404584 \\ \mathrm{C} & -0.305804 & 0.084082 & 1.082826 \\ \mathrm{C} & 0.928478 & -0.823875 & 0.847347 \\ \mathrm{C} & 1.992239 & -0.333128 & -0.106291 \\ \mathrm{C} & 0.018355 & -1.801216 & 0.164089 \\ \mathrm{C} & -2.020487 & -0.622687 & -0.824104 \\ \mathrm{C} & -1.230307 & -1.006502 & 0.396539 \\ \mathrm{O} & 3.023401 & -0.962532 & -0.275262 \\ \mathrm{O} & -1.198445 & 2.244098 & 0.667804 \\ \mathrm{H} & 2.471499 & 1.149936 & -1.627315 \\ \mathrm{H} & 0.534222 & 2.633675 & -1.183226 \\ \mathrm{H} & -0.569243 & 0.231205 & 2.134276 \\ \mathrm{H} & 1.430440 & -1.189213 & 1.755332 \\ \mathrm{H} & 0.230455 & -2.676677 & -0.443716 \\ \mathrm{H} & -4.131056 & -1.152607 & -0.328301 \\ \mathrm{H} & -1.444932 & -0.213656 & -1.663624 \\ \mathrm{H} & -1.911844 & -1.452899 & 1.138186\end{array}$


Int1-T1

SCF Energy: -535.795173584

ZPE-corrected Energy: -535.645808

$\Delta \mathrm{U}:-535.635946$

$\Delta \mathrm{H}:-535.635002$

$\Delta \mathrm{G}:-535.681877$

$S^{2}$ before (2.0105) and after higher multiplicity projection (2.0001)

Num. Imaginary Frequencies: 0

$\begin{array}{rrrr}\mathrm{C} & 0.484600 & -2.005440 & 0.128689 \\ \mathrm{C} & 0.101870 & 0.717287 & 1.842442 \\ \mathrm{C} & -0.853792 & -1.884526 & 0.156438 \\ \mathrm{C} & -1.552348 & -0.619153 & -0.178805 \\ \mathrm{C} & -0.767666 & 0.403150 & -0.954333 \\ \mathrm{C} & 0.771973 & 0.259362 & -0.994967 \\ \mathrm{C} & 1.385370 & -0.882645 & -0.231972 \\ \mathrm{C} & 0.903295 & 1.677623 & -0.442327 \\ \mathrm{C} & 0.184482 & 1.795374 & 0.898179 \\ \mathrm{C} & -0.605652 & 1.814686 & -0.387030 \\ \mathrm{O} & 2.572816 & -0.915904 & 0.049604 \\ \mathrm{O} & -2.720210 & -0.445642 & 0.129438 \\ \mathrm{H} & 0.978563 & -2.920295 & 0.452758 \\ \mathrm{H} & -1.490648 & -2.696960 & 0.502844 \\ \mathrm{H} & -1.196026 & 0.435486 & -1.963895 \\ \mathrm{H} & 1.147540 & 0.195601 & -2.024031 \\ \mathrm{H} & 1.648230 & 2.407831 & -0.742668 \\ \mathrm{H} & 0.712534 & 0.349908 & 2.657231 \\ \mathrm{H} & 0.290009 & 2.799855 & 1.319302 \\ \mathrm{H} & -1.223820 & 2.666634 & -0.651773\end{array}$




\begin{tabular}{|c|c|c|c|}
\hline \\
\hline \multicolumn{4}{|c|}{ Int2-S0opt } \\
\hline \multicolumn{4}{|c|}{ ZPE-corrected Energy: -535.779063} \\
\hline \multicolumn{4}{|c|}{$\Delta \mathrm{U}:-535.771289$} \\
\hline \multicolumn{4}{|c|}{$\Delta \mathrm{H}:-535.770345$} \\
\hline \multicolumn{4}{|c|}{$\Delta \mathrm{G}:-535.811871$} \\
\hline \multicolumn{4}{|c|}{ Num. Imaginary Frequencies: 0} \\
\hline $\mathrm{C}$ & -0.000539 & 1.422236 & 1.079069 \\
\hline $\mathrm{C}$ & -0.757112 & 1.754894 & -0.172686 \\
\hline $\mathrm{C}$ & -0.784966 & 0.485775 & -1.038879 \\
\hline $\mathrm{C}$ & 0.783888 & 0.486246 & -1.039058 \\
\hline $\mathrm{C}$ & 0.754986 & 1.755525 & -0.172746 \\
\hline $\mathrm{C}$ & 1.433181 & -0.675117 & -0.337471 \\
\hline $\mathrm{C}$ & 0.770595 & -1.054276 & 0.931386 \\
\hline $\mathrm{C}$ & -0.768460 & -1.055301 & 0.930931 \\
\hline $\mathrm{C}$ & -1.432647 & -0.676579 & -0.337271 \\
\hline $\mathrm{O}$ & -2.436753 & -1.233783 & -0.745967 \\
\hline $\mathrm{O}$ & 2.437525 & -1.231544 & -0.746899 \\
\hline $\mathrm{C}$ & 0.000403 & 0.024729 & 1.643665 \\
\hline $\mathrm{H}$ & -1.454606 & 2.583381 & -0.242857 \\
\hline $\mathrm{H}$ & -1.213116 & 0.623639 & -2.033884 \\
\hline $\mathrm{H}$ & -1.257059 & -1.833414 & 1.510925 \\
\hline $\mathrm{H}$ & 1.260284 & -1.832541 & 1.510374 \\
\hline $\mathrm{H}$ & 1.211843 & 0.624332 & -2.034075 \\
\hline $\mathrm{H}$ & 1.451824 & 2.584574 & -0.243812 \\
\hline $\mathrm{H}$ & -0.001107 & 2.212186 & 1.825945 \\
\hline $\mathrm{H}$ & -0.000205 & -0.048335 & 2.728674 \\
\hline
\end{tabular}


Int2-T1

SCF Energy: -535.846201369

ZPE-corrected Energy: -535.694483

$\Delta \mathrm{U}:-535.685611$

$\Delta \mathrm{H}:-535.684667$

$\Delta \mathrm{G}:-535.729296$

$S^{2}$ before (2.0182) and after higher multiplicity projection (2.0002)

Num. Imaginary Frequencies: 0

$\begin{array}{rrrr}\mathrm{C} & -0.738742 & 1.422179 & 0.999907 \\ \mathrm{C} & -1.068569 & 1.569167 & -0.476318 \\ \mathrm{C} & -0.607956 & 0.279706 & -1.183086 \\ \mathrm{C} & 0.865052 & 0.657763 & -0.814917 \\ \mathrm{C} & 0.333233 & 1.836020 & 0.003641 \\ \mathrm{C} & 1.705505 & -0.373127 & -0.099761 \\ \mathrm{C} & 0.981502 & -1.302065 & 0.722792 \\ \mathrm{C} & -0.492333 & -1.134769 & 0.907582 \\ \mathrm{C} & -1.105114 & -0.964662 & -0.498454 \\ \mathrm{O} & -1.914959 & -1.737198 & -0.958760 \\ \mathrm{O} & 2.935588 & -0.406676 & -0.216588 \\ \mathrm{C} & -0.854180 & 0.124072 & 1.655082 \\ \mathrm{H} & -1.831262 & 2.250434 & -0.840795 \\ \mathrm{H} & -0.816186 & 0.238699 & -2.254433 \\ \mathrm{H} & -0.933480 & -2.013189 & 1.386780 \\ \mathrm{H} & 1.523445 & -2.116536 & 1.199474 \\ \mathrm{H} & 1.405775 & 1.012295 & -1.697308 \\ \mathrm{H} & 0.845664 & 2.786283 & 0.122757 \\ \mathrm{H} & -1.081878 & 2.269971 & 1.590471 \\ \mathrm{H} & -1.387504 & 0.037341 & 2.597027\end{array}$


Int3-T1

SCF Energy: -535.846569319

ZPE-corrected Energy: -535.695381

$\Delta \mathrm{U}:-535.686192$

$\Delta \mathrm{H}:-535.685248$

$\Delta \mathrm{G}:-535.730570$

$S^{2}$ before (2.0167) and after higher multiplicity projection (2.0002)

Num. Imaginary Frequencies: 0

$\begin{array}{rrrr}\text { C } & -0.974608 & 1.367647 & 1.205889 \\ \mathrm{C} & -0.529777 & 1.720136 & -0.185259 \\ \mathrm{C} & -0.473317 & 0.491425 & -1.155538 \\ \mathrm{C} & 1.076450 & 0.449067 & -0.957116 \\ \mathrm{C} & 0.972451 & 1.790114 & -0.290210 \\ \mathrm{C} & 1.597509 & -0.618692 & 0.001731 \\ \mathrm{C} & 0.651799 & -1.362876 & 0.793862 \\ \mathrm{C} & -0.817424 & -1.090015 & 0.747640 \\ \mathrm{C} & -1.209923 & -0.711372 & -0.668067 \\ \mathrm{O} & -2.043053 & -1.321842 & -1.306277 \\ \mathrm{O} & 2.813475 & -0.803779 & 0.107311 \\ \mathrm{C} & -1.099989 & 0.110118 & 1.644453 \\ \mathrm{H} & -1.092660 & 2.581342 & -0.570081 \\ \mathrm{H} & -0.765605 & 0.742122 & -2.177633 \\ \mathrm{H} & -1.396282 & -1.962168 & 1.067980 \\ \mathrm{H} & 1.056851 & -2.091025 & 1.495075 \\ \mathrm{H} & 1.677942 & 0.393001 & -1.874034 \\ \mathrm{H} & 1.710333 & 2.373479 & 0.254430 \\ \mathrm{H} & -1.147079 & 2.194819 & 1.893363 \\ \mathrm{H} & -1.365895 & -0.099921 & 2.678317\end{array}$


TS1-T1

SCF Energy: -535.723092415

ZPE-corrected Energy: -535.580059

$\Delta \mathrm{U}:-535.569188$

$\Delta \mathrm{H}:-535.568243$

$\Delta \mathrm{G}:-535.617667$

$S^{2}$ before (2.0190) and after higher multiplicity projection (2.0002)

Num. Imaginary Frequencies: 1

Imaginary Frequency: -652.2540

$\begin{array}{lrrr}\mathrm{C} & 1.344399 & -1.391300 & 0.694363 \\ \mathrm{C} & -2.594444 & 0.382757 & 1.153786 \\ \mathrm{C} & 0.061991 & -1.816700 & 0.332709 \\ \mathrm{C} & -0.801636 & -1.119627 & -0.538740 \\ \mathrm{C} & -0.287697 & 0.153880 & -1.188547 \\ \mathrm{C} & 1.157869 & 0.621064 & -0.833273 \\ \mathrm{C} & 1.968214 & -0.216530 & 0.221872 \\ \mathrm{C} & 0.568293 & 1.828133 & -0.243166 \\ \mathrm{C} & -1.758772 & 1.289246 & 1.236573 \\ \mathrm{C} & -0.754035 & 1.455420 & -0.555573 \\ \mathrm{O} & 3.083198 & 0.223059 & 0.524379 \\ \mathrm{O} & -1.991766 & -1.435576 & -0.770473 \\ \mathrm{H} & 1.941074 & -1.993653 & 1.377690 \\ \mathrm{H} & -0.334749 & -2.734647 & 0.764022 \\ \mathrm{H} & -0.520612 & 0.107178 & -2.260045 \\ \mathrm{H} & 1.851668 & 0.786979 & -1.667283 \\ \mathrm{H} & 1.004069 & 2.680609 & 0.273093 \\ \mathrm{H} & -3.211961 & -0.458320 & 0.903542 \\ \mathrm{H} & -1.319753 & 2.098354 & 1.791844 \\ \mathrm{H} & -1.566280 & 2.095574 & -0.894119\end{array}$


TS2-T1

SCF Energy: -535.768996637

ZPE-corrected Energy: -535.622226

$\Delta \mathrm{U}:-535.612337$

$\Delta \mathrm{H}:-535.611393$

$\Delta \mathrm{G}:-535.658443$

$S^{2}$ before (2.0488) and after higher multiplicity projection (2.0005)

Num. Imaginary Frequencies: 1

Imaginary Frequency: -594.0529

$\begin{array}{lrrr}\mathrm{C} & -1.230242 & 1.663262 & 0.243969 \\ \mathrm{C} & 0.593533 & -0.796558 & 1.977163 \\ \mathrm{C} & 0.052391 & 2.054852 & 0.148946 \\ \mathrm{C} & 1.139844 & 1.149514 & -0.295094 \\ \mathrm{C} & 0.729740 & -0.100893 & -1.016189 \\ \mathrm{C} & -0.763417 & -0.509379 & -0.988749 \\ \mathrm{C} & -1.682851 & 0.293709 & -0.104151 \\ \mathrm{C} & -0.328613 & -1.889211 & -0.577691 \\ \mathrm{C} & 0.836415 & -1.694355 & 0.994958 \\ \mathrm{C} & 1.110025 & -1.492998 & -0.460035 \\ \mathrm{O} & -2.763319 & -0.125834 & 0.270765 \\ \mathrm{O} & 2.310416 & 1.431568 & -0.096787 \\ \mathrm{H} & -1.993463 & 2.320232 & 0.658546 \\ \mathrm{H} & 0.369445 & 3.043457 & 0.477941 \\ \mathrm{H} & 1.098381 & -0.009239 & -2.044963 \\ \mathrm{H} & -1.228150 & -0.488963 & -1.987684 \\ \mathrm{H} & -0.819277 & -2.854600 & -0.614160 \\ \mathrm{H} & 0.612604 & 0.262203 & 2.186483 \\ \mathrm{H} & 0.891698 & -2.747495 & 1.286181 \\ \mathrm{H} & 1.951023 & -2.039120 & -0.892937\end{array}$


TS3-T1

SCF Energy: -535.788029560

ZPE-corrected Energy: -535.638838

$\triangle \mathrm{U}:-535.629893$

$\Delta \mathrm{H}:-535.628949$

$\Delta \mathrm{G}:-535.673838$

$S^{2}$ before (2.0477) and after higher multiplicity projection (2.0006)

Num. Imaginary Frequencies: 1

Imaginary Frequency: -293.9079

$\begin{array}{lrrr}\mathrm{C} & 1.119011 & -1.506759 & 0.476893 \\ \mathrm{C} & -0.795938 & 0.235605 & 1.804335 \\ \mathrm{C} & -0.236306 & -1.673685 & 0.473275 \\ \mathrm{C} & -1.151141 & -0.983141 & -0.469131 \\ \mathrm{C} & -0.634252 & 0.267962 & -1.124315 \\ \mathrm{C} & 0.836609 & 0.649003 & -0.816268 \\ \mathrm{C} & 1.752176 & -0.355744 & -0.166094 \\ \mathrm{C} & 0.331205 & 1.832821 & 0.018569 \\ \mathrm{C} & -0.717513 & 1.460585 & 1.048925 \\ \mathrm{C} & -1.089136 & 1.556855 & -0.420910 \\ \mathrm{O} & 2.964789 & -0.182734 & -0.134588 \\ \mathrm{O} & -2.270950 & -1.417683 & -0.690370 \\ \mathrm{H} & 1.766034 & -2.159425 & 1.059452 \\ \mathrm{H} & -0.694424 & -2.494483 & 1.024712 \\ \mathrm{H} & -0.878129 & 0.246164 & -2.190263 \\ \mathrm{H} & 1.337076 & 1.033758 & -1.711114 \\ \mathrm{H} & 0.844137 & 2.787350 & 0.094930 \\ \mathrm{H} & -1.513723 & -0.095345 & 2.545951 \\ \mathrm{H} & -1.045686 & 2.345536 & 1.602983 \\ \mathrm{H} & -1.854291 & 2.238776 & -0.778653\end{array}$


TS4-T1

SCF Energy: -535.786102178

ZPE-corrected Energy: -535.639217

$\triangle \mathrm{U}:-535.629419$

$\Delta \mathrm{H}:-535.628475$

$\Delta \mathrm{G}:-535.675263$

$S^{2}$ before (2.0494) and after higher multiplicity projection (2.0010)

Num. Imaginary Frequencies: 1

Imaginary Frequency: -228.1906

$\begin{array}{lrrr}\mathrm{C} & -1.340651 & 1.408398 & 1.071737 \\ \mathrm{C} & -0.743823 & 1.618388 & -0.293344 \\ \mathrm{C} & -0.446961 & 0.332874 & -1.144674 \\ \mathrm{C} & 1.074439 & 0.543887 & -0.912274 \\ \mathrm{C} & 0.744486 & 1.851923 & -0.256308 \\ \mathrm{C} & 1.770800 & -0.409585 & 0.043607 \\ \mathrm{C} & 0.961699 & -1.413484 & 0.740834 \\ \mathrm{C} & -0.364942 & -1.591318 & 0.498040 \\ \mathrm{C} & -1.056716 & -0.938199 & -0.637887 \\ \mathrm{O} & -2.066736 & -1.422205 & -1.123471 \\ \mathrm{O} & 2.970889 & -0.299022 & 0.253541 \\ \mathrm{C} & -1.482018 & 0.253927 & 1.683876 \\ \mathrm{H} & -1.329647 & 2.371739 & -0.839746 \\ \mathrm{H} & -0.746678 & 0.474605 & -2.186530 \\ \mathrm{H} & -0.925462 & -2.380928 & 0.996349 \\ \mathrm{H} & 1.481368 & -2.000168 & 1.496138 \\ \mathrm{H} & 1.691397 & 0.602545 & -1.820627 \\ \mathrm{H} & 1.359481 & 2.547178 & 0.308330 \\ \mathrm{H} & -1.645059 & 2.322858 & 1.597247 \\ \mathrm{H} & -1.816503 & -0.108875 & 2.646637\end{array}$


TS5-T1

SCF Energy: -535.829293480

ZPE-corrected Energy: -535.679061

$\Delta \mathrm{U}:-535.670450$

$\Delta \mathrm{H}:-535.669506$

$\Delta \mathrm{G}:-535.713653$

$S^{2}$ before (2.0387) and after higher multiplicity projection (2.0007)

Num. Imaginary Frequencies: 1

Imaginary Frequency: -642.8197

$\begin{array}{lrrr}\mathrm{C} & -0.881566 & 1.371055 & 1.092052 \\ \mathrm{C} & -0.952543 & 1.623784 & -0.366249 \\ \mathrm{C} & -0.615026 & 0.334814 & -1.162942 \\ \mathrm{C} & 0.903864 & 0.608233 & -0.888984 \\ \mathrm{C} & 0.500686 & 1.868168 & -0.160725 \\ \mathrm{C} & 1.673879 & -0.438167 & -0.101552 \\ \mathrm{C} & 0.899316 & -1.336961 & 0.711660 \\ \mathrm{C} & -0.575379 & -1.126725 & 0.876598 \\ \mathrm{C} & -1.154680 & -0.904374 & -0.516830 \\ \mathrm{O} & -1.967159 & -1.649581 & -1.021713 \\ \mathrm{O} & 2.905782 & -0.499570 & -0.163991 \\ \mathrm{C} & -0.815259 & 0.128845 & 1.692356 \\ \mathrm{H} & -1.681600 & 2.341909 & -0.745474 \\ \mathrm{H} & -0.892195 & 0.381637 & -2.217873 \\ \mathrm{H} & -1.050817 & -2.000260 & 1.334022 \\ \mathrm{H} & 1.417610 & -2.148235 & 1.219847 \\ \mathrm{H} & 1.464007 & 0.812099 & -1.810952 \\ \mathrm{H} & 1.062658 & 2.738008 & 0.159120 \\ \mathrm{H} & -0.996545 & 2.251650 & 1.721581 \\ \mathrm{H} & -0.731860 & 0.044365 & 2.773057\end{array}$




\subsection{Exo approach}

Int1-S0

SCF Energy: -535.798305079

ZPE-corrected Energy: -535.648135

$\Delta \mathrm{U}:-535.638270$

$\Delta \mathrm{H}:-535.637326$

$\Delta \mathrm{G}:-535.683967$

Num. Imaginary Frequencies: 0

$\begin{array}{lrrr}\text { C } & -1.504540 & 0.735170 & 0.584829 \\ \mathrm{C} & -1.503878 & -0.736732 & 0.584732 \\ \mathrm{C} & -0.026988 & -0.772595 & 0.992499 \\ \mathrm{C} & -0.027700 & 0.772367 & 0.992556 \\ \mathrm{C} & 0.936262 & 1.487475 & 0.084143 \\ \mathrm{C} & 1.981889 & 0.673273 & -0.575297 \\ \mathrm{C} & 1.982470 & -0.671574 & -0.575404 \\ \mathrm{C} & 0.937566 & -1.486750 & 0.083991 \\ \mathrm{O} & 0.866192 & 2.689894 & -0.101376 \\ \mathrm{O} & 0.868526 & -2.689211 & -0.101664 \\ \mathrm{H} & -2.270260 & 1.445597 & 0.877243 \\ \mathrm{H} & -2.268954 & -1.447866 & 0.877097 \\ \mathrm{H} & 0.084588 & -1.190574 & 1.999710 \\ \mathrm{H} & 0.083407 & 1.190363 & 1.999819 \\ \mathrm{H} & 2.741625 & 1.238173 & -1.112932 \\ \mathrm{H} & 2.742703 & -1.235727 & -1.113127 \\ \mathrm{C} & -1.629415 & -0.000848 & -0.782724 \\ \mathrm{C} & -2.912143 & -0.000416 & -1.378527 \\ \mathrm{H} & -0.744825 & -0.000509 & -1.425564 \\ \mathrm{H} & -3.647166 & -0.001134 & -0.542718\end{array}$


TS2-S0

SCF Energy: -535.770810437

ZPE-corrected Energy: -535.623969

$\Delta \mathrm{U}:-535.613682$

$\Delta \mathrm{H}:-535.612738$

$\Delta \mathrm{G}:-535.660065$

Num. Imaginary Frequencies: 1

Imaginary Frequency: -423.6581

$\begin{array}{rrrr}\mathrm{C} & -1.567747 & 0.049750 & 0.763715 \\ \mathrm{C} & -1.060447 & -1.251362 & 0.857833 \\ \mathrm{C} & 0.361869 & -0.801823 & 1.052675 \\ \mathrm{C} & -0.187264 & 0.646722 & 1.089282 \\ \mathrm{C} & 1.374304 & -1.150442 & -0.016308 \\ \mathrm{C} & 2.005090 & -0.026427 & -0.742676 \\ \mathrm{C} & 1.568220 & 1.242968 & -0.666652 \\ \mathrm{C} & 0.406006 & 1.662009 & 0.150435 \\ \mathrm{O} & 1.671582 & -2.305965 & -0.256969 \\ \mathrm{O} & -0.039538 & 2.793239 & 0.071395 \\ \mathrm{H} & -2.517786 & 0.475533 & 1.069795 \\ \mathrm{H} & -1.516533 & -2.234715 & 0.824623 \\ \mathrm{H} & -0.179335 & 1.078466 & 2.096902 \\ \mathrm{H} & 0.784987 & -1.136375 & 2.011783 \\ \mathrm{H} & 2.035793 & 2.035860 & -1.247912 \\ \mathrm{H} & 2.839518 & -0.299201 & -1.386557 \\ \mathrm{C} & -1.707222 & -0.261277 & -0.962863 \\ \mathrm{C} & -2.868258 & -0.576146 & -1.453135 \\ \mathrm{H} & -0.762364 & -0.002191 & -1.430681 \\ \mathrm{H} & -3.687941 & -1.019402 & -0.887195\end{array}$




\section{References}

1. Gan, X., Jiang, W., Wang, W. \& Hu, L. An approach to 3,6-disubstituted 2,5-dioxybenzoquinones via two sequential Suzuki couplings. Three-step synthesis of leucomelone. Org. Lett. 11, 589-592 (2009).

2. APEX3 Version 2018.1-0 (Bruker AXS Inc., 2018).

3. SAINT Version 8.38A (Bruker AXS Inc., 2016).

4. SADABS Version 2016/2 Krause, L., Herbst-Irmer, R., Sheldrick, G. M. \& Stalke, D. Comparison of silver and molybdenum microfocus X-ray sources for single-crystal structure determination. J. Appl. Crystallogr. 48, 3-10 (2015).

5. Dolomanov, O. V., Bourhis, L. J., Gildea, R. J., Howard, J. A. K. \& Puschmann, H. OLEX2: A complete structure solution, refinement and analysis program. J. Appl. Crystallogr. 42, 339-341 (2009).

6. SHELXT Version 2014/5 and SHELXL Version 2017/1 Sheldrick, G. M. SHELXT - Integrated space-group and crystal-structure determination. Acta Crystallogr. Sect. A Found. Crystallogr. 71, 3-8 (2015).

7. Spek, A. L. Single-crystal structure validation with the program PLATON. J. Appl. Crystallogr. 36, 7-13 (2003).

8. Wei, Y., Tinoco, A., Steck, V., Fasan, R. \& Zhang, Y. Cyclopropanations via Heme Carbenes: Basic Mechanism and Effects of Carbene Substituent, Protein Axial Ligand, and Porphyrin Substitution. J. Am. Chem. Soc. 140, 16491662 (2018).

9. Yan Zhao and Donald G. Truhlar, "The M06 suite of density functionals for main group thermochemistry, thermochemical kinetics, noncovalent interactions, excited states, and transition elements: two new functionals and systematic testing of four M06-class functionals and 12 other functionals", Theor. Chem. Acc. 120, 215- 241 (2008).

10. Jacopo Tomasi, Benedetta Mennucci, and Roberto Cammi, "Quantum mechanical continuum solvation models", Chem. Rev. 105, 2999-3094 (2005).

11. M. J. Frisch, G. W. Trucks, H. B. Schlegel, G. E. Scuseria, M. A. Robb, J. R. Cheeseman, G. Scalmani, V. Barone, G. A. Petersson, H. Nakatsuji, X. Li, M. Car- icato, A. V. Marenich, J. Bloino, B. G. Janesko, R. Gomperts, B. Mennucci, H. P. Hratchian, J. V. Ortiz, A. F. Izmaylov, J. L. Sonnenberg, D. Williams-Young, F. Ding, F. Lipparini, F. Egidi, J. Goings, B. Peng, A. Petrone, T. Henderson, D. Ranas- inghe, V. G. Zakrzewski, J. Gao, N. Rega, G. Zheng, W. Liang, M. Hada, M. Ehara, K. Toyota, R. Fukuda, J. Hasegawa, M. Ishida, T. Nakajima, Y. Honda, O. Kitao, H. Nakai, T. Vreven, K. Throssell, J. A. Montgomery, Jr., J. E. Peralta, F. Ogliaro, M. J. Bearpark, J. J. Heyd, E. N. Brothers, K. N. Kudin, V. N. Staroverov, T. A. Keith, R. Kobayashi, J. Normand, K. Raghavachari, A. P. Rendell, J. C. Burant, S. S. Iyengar, J. Tomasi, M. Cossi, J. M. Millam, M. Klene, C. Adamo, R. Cammi, J. W. Ochterski, R. L. Martin, K. Morokuma, O. Farkas, J. B. Foresman, and D. J. Fox, "Gaussian 16 Revision C.01" (2016), Gaussian Inc. Wallingford CT.

12. Rü diger Bauernschmitt and Reinhart Ahlrichs, "Stability analysis for solutions of the closed shell Kohn Sham equation", J. Chem. Phys. 104, 9047-9052 (1996). 